\title{
Urbanization and prevalence of type 2 diabetes in Southern Asia: A systematic analysis
}

\author{
Arsalan Cheema, Davies Adeloye, \\ Simrita Sidhu, Devi Sridhar, \\ Kit Yee Chan
}

Centre for Population Health Sciences and the World Health Organization's Collaboration Centre for Population Health Research and Training, The University of Edinburgh Medical School, Edinburgh, Scotland, UK

\section{Correspondence to:}

\section{Dr Kit Yee Chan}

Centre for Population Health Sciences

The University of Edinburgh Medical School

Teviot Place

Edinburgh, EH8 9AG

Scotland, UK

k.chan@ed.ac.uk

\begin{abstract}
Background Diabetes mellitus is one of the diseases considered to be the main constituents of the global non-communicable disease (NCD) pandemic. Despite the large impact that NCDs are predicted to have, particularly in developing countries, estimates of disease burden are sparse and inconsistent. This systematic review transparently estimates prevalence of type 2 diabetes mellitus in Southern Asia, its association with urbanization and provides insight into the policy challenges facing the region.
\end{abstract}

Methods The databases Medline and PubMed were searched for population-based studies providing estimates of diabetes prevalence in the Southern Asia region. Studies using WHO diagnostic criteria of fasting plasma glucose (FPG) $\geq 7.0 \mathrm{mmol} / \mathrm{L}$ and/or 2-hour plasma glucose $(2 \mathrm{hPG}) \geq 11.1 \mathrm{mmol} / \mathrm{L}$ were included. Data from eligible studies was extracted into bubble graphs, and trend lines were applied to UNPD figures to estimate age-specific prevalence in the regional population. Estimates specific to sex, area of residency, and diagnostic method were compared and trends analysed.

Results A total of 151 age-specific prevalence estimates were extracted from 39 studies. Diabetes prevalence was estimated to be $7.47 \%$ for 2005 and $7.60 \%$ for 2010 . Prevalence was strongly associated with increased age, male gender and urban residency $(P<0.001)$.

Conclusion Diabetes prevalence in Southern Asia is high and predicted to increase in the future as life expectancy rises and the region continues to urbanise. Countries in this region need to improve NCD surveillance and monitoring so policies can be informed with the best evidence. Programs for prevention need to be put in place, and health system capacity and access needs to be assessed and increased to deal with the predicted rise in NCD prevalence.

In recent years the issue of non-communicable diseases (NCDs) has been identified as a pressing concern that has come to the forefront of international policy discussion. NCDs are the leading causes of death and disability worldwide [1]. It was estimated that 33 million deaths in 2008 occurred from NCDs, accounting for almost two-thirds of all deaths for that year [2]. In addition, estimates suggest that these may increase further to a projected 52 million deaths by 2030, nearly five times as many deaths as projected for communicable diseases [3]. Type 2 diabetes mellitus (DM), 
along with cardiovascular disease, cancers, and chronic respiratory diseases, are considered four primary constituent diseases of the global NCD pandemic [3,4]. Importantly, the main risk factors for these diseases are modifiable and these diseases are heavily influenced by lifestyle and behaviour [5]. Shared risk factors between these diseases - such as eating an unhealthy diet high in saturated fat and sugar, a lack of physical activity, and tobacco smoking - account for over two-thirds of new NCD cases and increase the risk of exacerbations in those who already have these diseases [5]. These risk factors and resulting diseases are not limited to high-income countries - a disproportionate NCD burden is borne by developing countries. Over $80 \%$ of diabetes and cardiovascular deaths worldwide occur in low- and middle-income countries (LMICs), and many of the risk factors for these NCDs are associated with the country development process through globalisation and urbanisation [3]. The prevalence of the common NCDs increases with advancing age, so as life expectancy in LMICs increases the burden of NCDs is also expected to rise.

Non-communicable diseases are more likely to affect people who are socioeconomically disadvantaged, furthering health inequalities [6]. This can be due to contextual factors relating to the society and place in which people live in addition to behavioural factors. In LMICs, diabetes and its risk factors are associated with lower education levels [3]. The higher burden of NCDs poses additional problems for populations of developing countries that have lower levels of educational achievement and income. Limited health care capacities and lack of social protection for large parts of the population mean that treatment and support for NCDs is often unavailable or catastrophically expensive $[5,7]$. In addition, NCDs have significant socioeconomic effects. Nearly a third of NCD deaths in LMICs occur below the age of 60 [1]. These deaths at economically and socially productive ages have much wider consequences for these developing countries, with the loss of productivity and health system expenditure becoming major barriers for national economic development and progress [5,8]. On individual or household levels, the sustained nature of NCDs and resulting disabilities can lead to difficulties in working or seeking employment. Additionally, the long-term care that NCDs require and the high cost of health care in many developing countries have major impacts on household income, potentially leading to vicious cycles of poverty and illness [5]. The overall economic cost of NCDs cannot be understated: in India in 2004-2005, NCD health care expenditure and total income lost due to these diseases was estimated to amount to $1 \%$ of its massive economy [3].

Despite the serious implications of the global NCD burden, it is only recently that determined policy action has been seen. The UN High-Level Meeting on NCDs in 2011 led the way for an international response, providing guidance on how to integrate NCD prevention and control across sectors and at all levels of government [1,3]. Furthermore, monitoring and surveillance capabilities of several highburden countries have shown an increased capacity in recent years [2]. However, many of the recommended changes - such as health care system reform towards sustainable universal care, and integration of NCD prevention into multi-sectoral responses - may take several years to implement, particularly in the LMICs that bear the brunt of the global NCD burden. Meanwhile it is essential that these regions have reliable estimates of NCD burden to inform policy decisions with relevant evidence and help set appropriate health care and research priorities [9]. Transparent, up-to-date estimates of NCD burden allow monitoring of the diseases as well as evaluation of current policies, and are vital tools for planning policies and interventions to tackle the global NCD pandemic. This paper will attempt to address part of this need by carrying out a systematic literature review to estimate the prevalence of type $2 \mathrm{DM}$ in Southern Asia.

Box 1 briefly reviews approaches to diagnosis and known risk factors for type 2 diabetes [10-18]. Table 1 displays current WHO diagnostic criteria for venous plasma for fasting plasma glucose (FPG) and oral glucose tolerance test (OGTT). Diagnosis can be made through the use of either test alone or together. Specific values for capillary measurements and whole blood have been provided in previous WHO publications as well [12]. In terms of geographic focus of this study, the UN's Southern Asia region is comprised of nine countries [19]. General information regarding each country is given in Table 2, sourced from the World Bank online database [20]. The majority of Southern Asian countries are low or low-middle income countries [20]. The total population of the Southern Asia region comprises approximately $25 \%$ of the world total population [20]. India was estimated to have the highest number of diabetic adults in 2000 [21] and 2010 [22], and both these studies predicted it would continue to have the highest number of diabetic adults by 2030. Pakistan and Bangladesh were both estimated to be in the top ten as well. As such, an estimate of the diabetes prevalence for this region would provide a major insight into the global picture of diabetes burden.

This paper aims: (i) to contribute to the evidence base on type 2 diabetes mellitus in Southern Asia by systematically reviewing the relevant literature; (ii) to compare the prevalence estimates provided through different methods of diagnosing type 2 diabetes mellitus; (iii) to provide an assessment of the role of urbanization on the burden of Type 2 diabetes mellitus in the UN Southern Asia region based on the best available evidence; and (iv) to discuss the significance of the regional estimate and the implications it may have on public health policy. 
Box 1 Type 2 diabetes - diagnosis and risk factors

Type 2 diabetes mellitus (DM) is a metabolic disease characterised by persistent hyperglycaemia and disturbed carbohydrate, protein, and fat metabolism. It may present with combinations of typical symptoms such as polydipsia (increased thirst), polyphagia (excessive hunger), polyuria (increased passage of urine), glycosuria (glucose in urine), lethargy, and weight loss. These symptoms reflect the underlying DM pathophysiology of peripheral insulin resistance combined with inadequate pancreatic insulin secretion [10]. Many diabetic patients may be asymptomatic but in the long term uncontrolled hyperglycaemia can lead to severe complications such as diabetic retinopathy, neuropathy and nephropathy. Type $2 \mathrm{DM}$ can be diagnosed through biochemical measurements even if there are no presenting symptoms [11].

Under World Health Organisation (WHO) guidelines, there are currently two main diagnostic tests used to diagnose DM - the Fasting Plasma Glucose (FPG) test and the Oral Glucose Tolerance Test (OGTT) [11]. FPG involves measuring the level of glucose in a fasting (.8 hours without food) patient's blood, often after an overnight fast. OGTT is also carried out on fasting patients and involves measurement of baseline blood glucose, followed by ingestion of $75 \mathrm{~g}$ anhydrous glucose, and a subsequent blood glucose measurement after two hours to determine the efficacy with which glucose has been eliminated from the patient's blood [12]. Although WHO has also recently advocated the measurement of glycatedhaemoglobin $\left(\mathrm{HbAl}{ }_{\mathrm{C}}\right)$ for diagnostic purposes [13], the stringent quality assurance tests required for its effective usage have limited its use in epidemiological studies to date. Additional notes on these diagnostic methods are provided in Online Supplementary Document.

The aetiology of type $2 \mathrm{DM}$ is complex and likely involves a host of different factors, many of which are not fully understood. Common risk factors in the general population include older age, being overweight or obese, hypertension, leading an inactive lifestyle, smoking, and consuming an energy-dense diet $[14,15]$. Several of these risk factors may be considered 'lifestyle' factors that are potentially modifiable. However, a strong genetic component is also implicated in Type 2 DM, with relatives of diabetics at increased risk of developing it themselves, and certain ethnic populations believed to have increased susceptibility to diabetes $[16,17]$. South Asians in particular have been found to possess adverse body fat patterning that that may predispose to insulin resistance [18], and have higher diabetes risk than Caucasians with equivalent body mass indices (BMI) [16]. This non-modifiable genetic susceptibility for South Asians means it is of even greater importance that policies address modifiable risk factors in order to tackle burgeoning diabetes prevalence in the region.

Table 1. World Health Organization's 2006 diagnostic criteria for type 2 diabetes

Fasting plasma glucose (FPG) $\geq 7.0 \mathrm{mmol} / \mathrm{L}(126 \mathrm{mg} / \mathrm{dl})$

and / or

2-hour plasma glucose (OGTT 2hPG) $\geq 11.1 \mathrm{mmol} / \mathrm{L}$ (200mg/dl)

FPG -fasting plasma glucose, OGTT -oral glucose tolerance test.

\section{METHODS}

A systematic literature search of published studies providing population-based prevalence estimates of type 2 diabetes mellitus in Southern Asia was carried out. The online databases Medline and PubMed were searched, using the OVID search form for the Medline database and the default search engine for PubMed. Search terms for Medline and
PubMed are given in Table 3 and Table 4 respectively. Both Medical Subject Headings (MeSH terms) and keywords were used for the Medline search. The Medline search was more focused due to OVID's Advanced Search feature, while the PubMed search was left broader in order to pick up a larger selection of studies. The final searches were carried out on 13 February 2013. Box 2 shows inclusion criteria, exclusion criteria and quality evaluation criteria.

\section{Study selection}

The literature search of online databases resulted in a total of 5653 studies: 1754 from Medline and 3899 from PubMed. After initial analysis of titles and abstracts, 402 studies were selected that matched inclusion and exclusion criteria. 51 duplicate studies were removed and full texts

Table 2. Southern Asia countries - selected characteristics

\begin{tabular}{|c|c|c|c|c|c|c|}
\hline Countay & $\begin{array}{l}\text { Population, } \times 1000 \\
(2011)\end{array}$ & $\begin{array}{l}\text { LIFE EXPECTANCY AT BIRTH } \\
(2010)\end{array}$ & $\begin{array}{l}\text { BNI PER CAPITA } \\
\text { (US\$) (2011) }\end{array}$ & $\begin{array}{l}\text { GDP US\$ GROWTH, \% } \\
\text { (2011) }\end{array}$ & $\begin{array}{l}\text { TOTAL EXPENDITURE ON HEALTH } \\
(\% \text { GDP) }(2011)\end{array}$ & $\begin{array}{l}\text { Physicians PeR } 1000 \\
\text { POPULATION (2011) }\end{array}$ \\
\hline Afghanistan & 35320 & 48 & 1140 & $+6 \%$ & 9.6 & 0.210 \\
\hline Bangladesh & 150394 & 69 & 940 & $+7 \%$ & 3.7 & 0.295 \\
\hline Bhutan & 738 & 67 & 5570 & $+6 \%$ & 4.1 & 0.023 \\
\hline India & 1241492 & 65 & 3590 & $+7 \%$ & 3.9 & 0.649 \\
\hline Iran & 74799 & 73 & $11420 \dagger$ & $+2 \% \dagger$ & $5.8 *$ & 0.890 \\
\hline Maldives & 320 & 77 & 7430 & $+7 \%$ & 8.5 & 1.595 \\
\hline Nepal & 30486 & 68 & 1260 & $+4 \%$ & 5.4 & $0.210 \dagger$ \\
\hline Pakistan & 176745 & 65 & 2870 & $+3 \%$ & 2.5 & 0.813 \\
\hline Sri Lanka & 20869 & 75 & 5520 & $+8 \%$ & 3.4 & 0.492 \\
\hline
\end{tabular}

GNI - gross national income, GDP - gross domestic product

*Data from 2009, most recent available data but likely inaccurate following punitive economic sanctions.

†Data from 2004. 
Table 3. OVID Medline search terms

\begin{tabular}{|c|c|c|}
\hline & No. OF STUDIES \\
\hline \multicolumn{2}{|c|}{1 Diabetes Mellitus, type 2/ } & 74709 \\
\hline 2 & "adult-onset diabetes".tw & 358 \\
\hline 3 & (diabetes adj2 type 2 ).tw & 54707 \\
\hline 4 & "non-insulin dependent diabetes".tw & 8297 \\
\hline 5 & NIDDM.tw & 6732 \\
\hline 6 & Diabetes Mellitus/ or Diabetes Mellitus, type 2/ & 155830 \\
\hline 7 & 1 OR 2 OR 3 OR 4 OR 5 OR 6 & 173525 \\
\hline 8 & exp morbidity/ or exp mortality/ & 563526 \\
\hline 9 & incidence.tw & 464724 \\
\hline 10 & (prevalen* or mortality or epidemiol*).tw & 961509 \\
\hline 11 & Epidemiology/ & 11218 \\
\hline 12 & "cost of illness"/ & 15625 \\
\hline 13 & (burden adj2 diseas*).tw & 7633 \\
\hline 14 & 8 OR 9 OR 10 OR 11 OR 12 OR 13 & 1586465 \\
\hline 15 & Bangladesh/ or Bhutan/ or India/ or Afghanistan/ or Iran/ or Nepal/ or Pakistan/ or "Sri Lanka"/ & 102952 \\
\hline 16 & Indian Ocean Islands/ & 568 \\
\hline 17 & (afghan* or bangladesh* or bengal* or bhutan* or iran* or india* or nepal* or pakistan* or maldiv* or srilanka $*$.tw & 122321 \\
\hline 18 & 15 OR 16 OR 17 & 161040 \\
\hline 19 & 7 AND 14 AND 18 & 1896 \\
\hline 20 & Limit 19 to (humans and yr="1980-Current") & 1754 resul \\
\hline
\end{tabular}

Box 2 Literature search: Inclusion criteria, exclusion criteria and quality evaluation criteria

\section{Inclusion criteria:}

- Population- or community-based study in a Southern Asian country providing prevalence estimates of type 2 diabetes mellitus based on primary data.

- All published study designs and all languages.

- Studies post-1980 with $\geq 200$ participants.

- Studies looking at adults ( $\geq 20$ years).

- Studies diagnosing diabetes through biochemical measurements.

\section{Exclusion criteria:}

- Studies investigating other forms of diabetes, such as gestational diabetes or diabetes insipidus.

- Hospital- or clinic-based studies.

- Studies diagnosing diabetes through self-reported questionnaires or symptoms only.

- Study populations specifically predisposed to diabetes, such as relatives of known diabetics.

- Studies investigating prevalence of complications in a diabetic cohort without commenting on actual prevalence of diabetes in area or community.

\section{Quality evaluation criteria:}

- Diabetes diagnosed through fasting plasma glucose (FPG) after $\geq 8$ hours fasting, and/or oral glucose tolerance test (OGTT) two hours after ingestion of $75 \mathrm{~g}$ anhydrous glucose or equivalent.

- Appropriate diagnostic criteria for diabetes - most recent WHO recommendations of $\mathrm{FPG} \geq 7.0 \mathrm{mmol} / \mathrm{L}$ and/or $2 \mathrm{hPG} \geq 11.1 \mathrm{mmol} / \mathrm{L}$ for venous plasma, or equivalent for other sample types. Stated whether blood samples were venous or capillary, and whether whole blood or plasma was analysed.

- Clearly defined population recruited through representative sampling methods.

- Description of how known diabetics were accounted for.
Table 4. PubMed search terms

SEARGH TERMS
Diabetes AND (Afghanistan OR Bangladesh OR 3899 results
Bhutan OR India OR Iran OR Maldives OR Nepal
OR Pakistan OR Sri Lanka) AND (Epidemiology
OR Incidence OR Prevalence OR Mortality)

of the remaining 351 studies were further analysed and quality assessed. 39 studies were included in the final analysis, including 2 papers identified through reference lists of other assessed studies. A visual summary of the study selection process is presented in Figure 1.

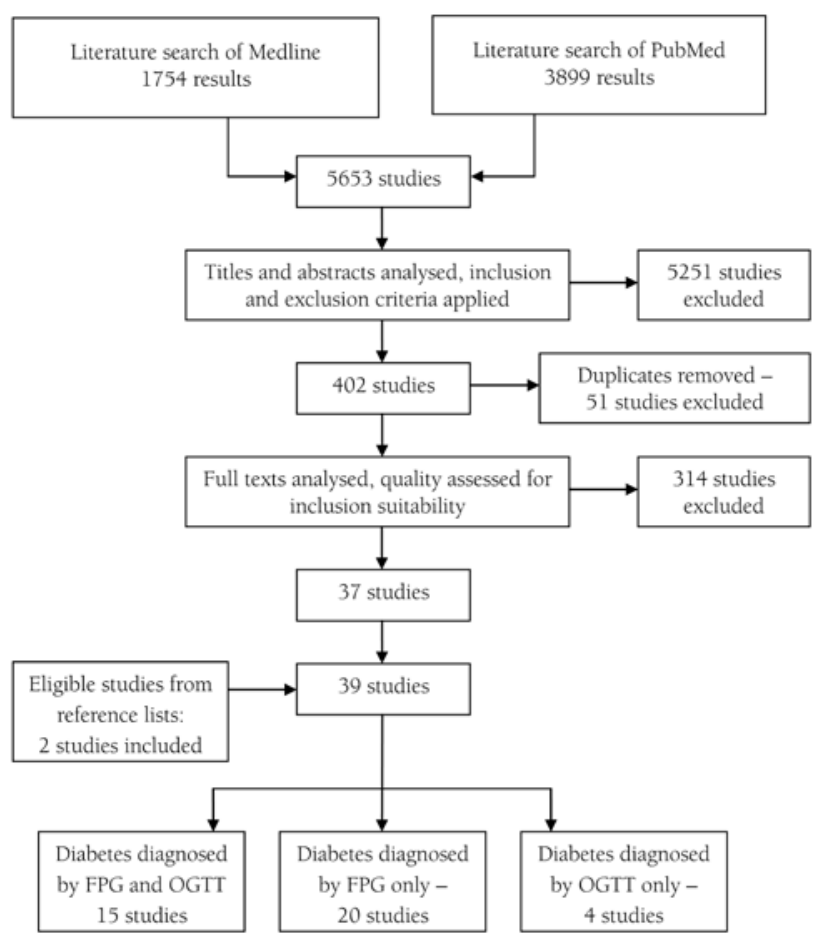

Figure 1. Study selection process. FPG -fasting plasma glucose, OGTT -oral glucose tolerance test. 


\section{Data extraction}

Titles and abstracts of all studies obtained through the database searches were evaluated. Inclusion and exclusion criteria were applied. Basic details of all studies such as title, authors, country, study year, year of publication, and sample size were extracted into an Excel spreadsheet for ease of full text evaluation. After initial extraction, full texts of the studies were analysed and assessed for quality criteria. Quality assessment information is presented in Online Supplementary Document. Studies for which full text was not available were requested through inter-library loans. Duplicate studies were identified through study locations and matching sample sizes, and were removed. In addition, the reference lists of the selected studies were examined for relevant papers not captured by the literature search. These new studies were subsequently evaluated and added to the spreadsheet.

Another Excel spreadsheet was created for eligible studies selected through full text analysis. All the above data was extracted in addition to data on method of diagnosis; diagnostic criteria; specific location of the study; whether the study described the surveyed area as urban, rural, mixed or none; age range of participants and mean age if provided; diabetes prevalence in sample; and sex-specific sample size, mean age, and diabetes prevalence. Many studies looked at several different cohorts in various areas, often for purpose of comparison. These multiple cohorts were recorded separately so that individual sample characteristics could be differentiated. Three separate sheets were created for studies depending on their method of diagnosing new diabetes: one for studies that diagnosed diabetes on the basis of both FPG and OGTT results; one for studies using only FPG results; and one for studies using only OGTT results. These spreadsheets were the basis for prevalence estimation.

\section{Data analysis}

To allow for comparison between studies, all reported prevalence estimates were converted to prevalence/1000 population through the equation:

Prevalence $=$ Number of diabetes cases $\times 1000 /$ Sample size

During data modelling, the mean age, sample size, and age-specific prevalence estimates (per 1000 population) of all selected studies were used to create bubble graphs representing the data. If this information was missing for particular cohorts, it was calculated from the data that was available, as detailed in Online Supplementary Document. Several bubble graphs were created: for overall prevalence, sex-specific prevalence, urban/rural prevalence, and prevalence for specific diagnostic methods

In order to calculate population prevalence estimates for the region, trend lines with the power function were com-

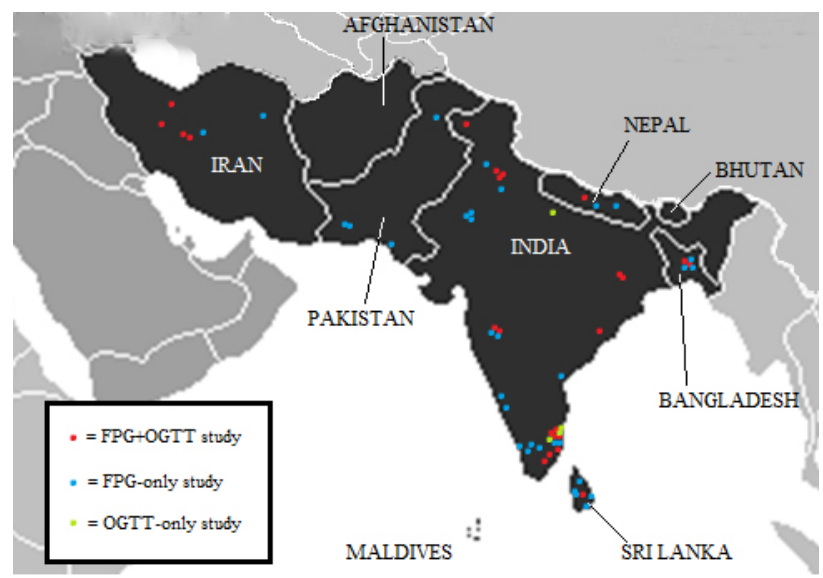

Figure 2. Approximate geographic locations of the study cohorts. FPG -fasting plasma glucose, OGTT -oral glucose tolerance test.

puted from the graphs to represent the relationship between age and prevalence for the selected data set. These were chosen because they had the highest $r-$ squared $\left(R^{2}\right)$ values for these graphs and therefore accounted for the highest fraction of variance in the data. Statistical significance ( $p$-values) of differences observed through any comparisons was derived directly from the model. The resulting equations for overall combined prevalence, total male prevalence and total female prevalence were applied to 2005 UNPD population estimates, the closest to the median study year of 2006 [23]. Prevalence results were multiplied by population figures for each age group, thereby giving an estimate of the total number of expected diabetes cases for each one. Totalling these up and taking a percentage of the total adult population allowed calculation of overall diabetes prevalence, for each sex separately and combined.

\section{Study characteristics}

Of the 39 studies included in the analysis, 15 studies diagnosed diabetes using both FPG and OGTT methods, 20 solely using FPG, and 4 studies using OGTT only. Several studies looked at more than one cohort when estimating diabetes prevalence, often for purposes of comparison. A total of 57 cohorts were investigated by the 39 studies (Figure 2). During analysis each different cohort was represented independently. Within these cohorts, age-specific prevalence estimates were represented individually if available. Table 5 provides an overview of study characteristics by country. Population estimates are based on 2005 UNPD data [23].

The country with the largest number of studies, of all types, was by far India. It was also the only country for which suitable OGTT-only studies were available. No suitable studies of any kind were found in the literature search for 
Table 5. Study characteristics by country

\begin{tabular}{|c|c|c|c|c|c|}
\hline Countriy & $\begin{array}{l}\text { PropoRtion of REeGONAL ADULt } \\
(\geq 20) \text { POPULation }(\%)\end{array}$ & $\begin{array}{l}\text { No. of FPG \& OGTT } \\
\text { COMBINED STUDIES }\end{array}$ & No. of FPG-ONIY STUdIES & $\begin{array}{l}\text { No. of OGTT-0NLY } \\
\text { STUDIES }\end{array}$ & Total NIo. OF STUDIES \\
\hline Afghanistan & 1.28 & 0 & 0 & 0 & 0 \\
\hline Bangladesh & 8.65 & 2 & 2 & 0 & 4 \\
\hline Bhutan & 0.04 & 0 & 0 & 0 & 0 \\
\hline India & 73.24 & 9 & 8 & 4 & 21 \\
\hline Iran & 4.78 & 2 & 2 & 0 & 4 \\
\hline Maldives & 0.02 & 0 & 0 & 0 & 0 \\
\hline Nepal & 1.53 & 1 & 2 & 0 & 3 \\
\hline Pakistan & 8.97 & 0 & 4 & 0 & 4 \\
\hline Sri Lanka & 1.49 & 1 & 2 & 0 & 3 \\
\hline Total & 100.00 & 15 & 20 & 4 & 39 \\
\hline
\end{tabular}

FPG - fasting plasma glucose, OGTT - oral glucose tolerance test

three countries: Afghanistan, Bhutan, and the Maldives. The remaining five countries had a mixture of FPG-only and FPG+OGTT combined studies, with the exception of Pakistan, for which only FPG studies were found. The mean study size was 5178, with samples ranging from 331 to 25969 participants. The median study year based on provided information was 2006 - while the earliest publication year was 1992, the earliest specified study year was 1998 and the most recent study year was 2009. More information about the study cohorts is given in Table 6 and Table 7, and additional information is provided in Online Supplementary Document.

\section{RESULTS}

Figure 3 displays the relationship between mean age of sample and overall diabetes prevalence (both sexes combined, all diagnostic methods). A total of 151 individual

Table 6. Cohort characteristics by country

\begin{tabular}{|c|c|c|c|c|}
\hline Country & $\begin{array}{l}\text { No. OF FPG \& } \\
\text { OGTT COMBINED } \\
\text { COHORTS }\end{array}$ & $\begin{array}{l}\text { Nlo. OF FPG- } \\
\text { onIY EOHORTS }\end{array}$ & $\begin{array}{l}\text { No. OF } \\
\text { OGTT-ONIY } \\
\text { COHORTS }\end{array}$ & $\begin{array}{l}\text { TOTAL Nlo. OF } \\
\text { COHORIS }\end{array}$ \\
\hline Afghanistan & 0 & 0 & 0 & 0 \\
\hline Bangladesh & 2 & 3 & 0 & 5 \\
\hline Bhutan & 0 & 0 & 0 & 0 \\
\hline India & 20 & 9 & 6 & 35 \\
\hline Iran & 2 & 2 & 0 & 4 \\
\hline Maldives & 0 & 0 & 0 & 0 \\
\hline Nepal & 1 & 2 & 0 & 3 \\
\hline Pakistan & 0 & 4 & 0 & 4 \\
\hline Sri Lanka & 1 & 5 & 0 & 6 \\
\hline Total & 26 & 25 & 6 & 57 \\
\hline
\end{tabular}

FPG - fasting plasma glucose, OGTT - oral glucose tolerance test

Table 7. General cohort characteristics

\begin{tabular}{|c|c|c|c|c|}
\hline Characteristic & $\begin{array}{l}\text { FPG \& OGTT } \\
\text { COMBINED } \\
\text { COHORTS }\end{array}$ & $\begin{array}{l}\text { FPG-ONIY } \\
\text { COHORTS }\end{array}$ & $\begin{array}{l}\text { OGTT-ONIY } \\
\text { COHORTS }\end{array}$ & Total \\
\hline Rural & 9 & 13 & 3 & 25 \\
\hline Urban & 15 & 7 & 3 & 25 \\
\hline Both/None & 2 & 5 & 0 & 7 \\
\hline Minimum size & 526 & 331 & 588 & \\
\hline Maximum size & 12514 & 25969 & 1213 & \\
\hline Mean size & 3580 & 4126 & 954 & \\
\hline
\end{tabular}

FPG - fasting plasma glucose, OGTT - oral glucose tolerance test

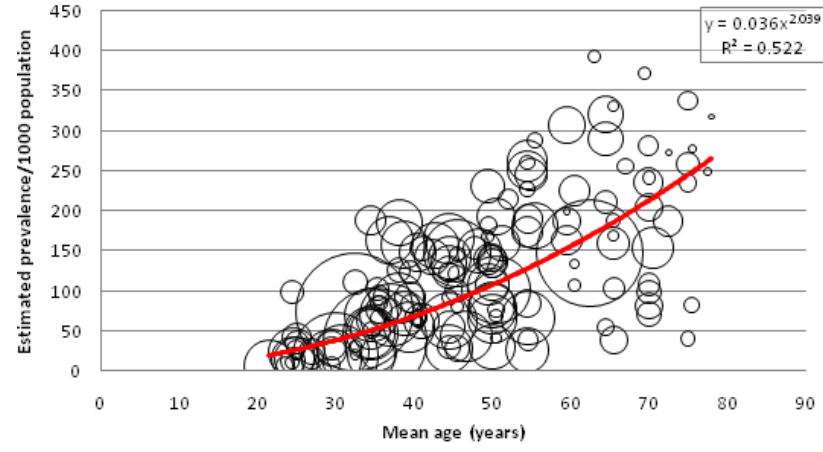

Figure 3. The relationship between crude prevalence of type 2 diabetes in Southern Asia and age.

data points for age-specific prevalence were plotted from 57 cohorts. 65 individual points were available from FPG and OGTT combined studies, 77 from FPG-only studies, and 9 from OGTT-only studies (Tables 8,9 and 10). Age-specific prevalence data are provided in Online Supplementary Document. Figure 3 shows a positive association between mean age and diabetes prevalence. Table 11 illustrates this association using the trend line equation derived from overall prevalence results and also displays population prevalence estimates based on 2005 UNPD estimates for the Southern Asia region. Based on these figures, the diabetes population prevalence for 2005 was estimated to be $7.47 \%$. The $50-54$ age group had the highest proportion of the burden at $11.31 \%$, and the 20 24 group had the lowest proportion of the burden at $4.64 \%$. A total of $51.3 \%$ of the burden was seen in those aged 50 or more. The diabetes burden increases with age until the ages of 50 and 54, after which it decreases. This is due to the population age structure in 2005 -diabetes prevalence per 1000 population is shown to increase continuously with age.

Table 12 shows the overall prevalence equation applied to 2010 UNPD population estimates, and Figure 4 compares the estimated numbers of diabetics between 2005 and 2010. A higher overall prevalence estimate is observed for 
Table 8. Individual study prevalence data: FPG+OGTT combined studies

\begin{tabular}{|c|c|c|c|c|c|c|c|c|c|c|c|c|}
\hline AUthors & Country & $\begin{array}{l}\text { URBAN/ } \\
\text { RURAL }\end{array}$ & $\begin{array}{l}\text { STUDY } \\
\text { SAMPLE } \\
\text { SIZE }\end{array}$ & $\begin{array}{c}\text { AGE } \\
\text { RANGE OF } \\
\text { PARTICI- } \\
\text { PANTS }\end{array}$ & $\begin{array}{c}\text { MEAN } \\
\text { AGE }\end{array}$ & $\begin{array}{c}\text { OVERALL } \\
\text { DIABETES } \\
\text { PREVALENCE } \\
\text { (1000 } \\
\text { POPULATION) }\end{array}$ & $\begin{array}{c}\text { Male } \\
\text { DIABETES } \\
\text { PREVALENCE } \\
\text { (V1000 } \\
\text { MEN) }\end{array}$ & $\begin{array}{c}\text { MEAN } \\
\text { AGE }\end{array}$ & $\begin{array}{l}\text { Size OF } \\
\text { GROUP }\end{array}$ & $\begin{array}{c}\text { FEMALE } \\
\text { DIABETES } \\
\text { PREVALENCE } \\
\text { (V1000 } \\
\text { WOMEN) }\end{array}$ & $\begin{array}{c}\text { Mean } \\
\text { aGE }\end{array}$ & $\begin{array}{l}\text { SIZE OF } \\
\text { GROUP }\end{array}$ \\
\hline Bhowmik et al (2012) [24] & Bangladesh & Rural & 2293 & $\geq 20$ & 50 & 79 & 90 & 43.9 & 842 & 71 & 40.4 & 1451 \\
\hline Rahim et al (2008) [25] & Bangladesh & Rural & 3954 & $\geq 20$ & 37.1 & 70 & 75 & 39 & 1592 & 67 & 35.9 & 2375 \\
\hline Nazir et al (2012) [26] & India & Urban & 2188 & $\geq 20$ & 38.7 & 158 & - & - & - & - & - & - \\
\hline Prasad et al (2012) [27] & India & Urban & 1178 & $20-80$ & 45.6 & 157 & 178 & 47 & 590 & 138 & 44.2 & 588 \\
\hline \multirow[t]{8}{*}{ Anjana et al (2011) [28] } & \multirow[t]{8}{*}{ India } & Urban & 1029 & $\geq 20$ & $50 *$ & 137 & - & - & - & - & - & - \\
\hline & & Rural & 2480 & $\geq 20$ & $50 *$ & 78 & - & - & - & - & - & - \\
\hline & & Urban & 1093 & $\geq 20$ & $50 *$ & 109 & - & - & - & - & - & - \\
\hline & & Rural & 2476 & $\geq 20$ & $50 *$ & 65 & - & - & - & - & - & - \\
\hline & & Urban & 840 & $\geq 20$ & $50 *$ & 135 & - & - & - & - & - & - \\
\hline & & Rural & 2051 & $\geq 20$ & $50 *$ & 30 & - & - & - & - & - & - \\
\hline & & Urban & 839 & $\geq 20$ & $50 *$ & 142 & - & - & - & - & - & - \\
\hline & & Rural & 2247 & $\geq 20$ & $50 *$ & 83 & - & - & - & - & - & - \\
\hline \multirow{2}{*}{ Deepa et al (2011) [29] } & \multirow{2}{*}{ India } & Urban & 526 & $\geq 20$ & 47.8 & 154 & - & - & - & - & - & - \\
\hline & & Urban & 596 & $\geq 20$ & 41.1 & 153 & - & - & - & - & - & - \\
\hline Ravikumar et al (2011) [30] & India & Urban & 2227 & $\geq 20$ & 42.7 & 157 & - & - & - & - & - & - \\
\hline \multirow[t]{3}{*}{ Ramachandran et al (2008) [31] } & \multirow[t]{3}{*}{ India } & Urban & 2192 & $\geq 20$ & 38.2 & 186 & 209 & $38.2 \dagger$ & 1053 & 167 & $38.2 \dagger$ & 1139 \\
\hline & & Urban & 2290 & $\geq 20$ & 36.8 & 164 & 171 & $36.8 \dagger$ & 988 & 159 & $36.8 \dagger$ & 1302 \\
\hline & & Rural & 2584 & $\geq 20$ & 38 & 92 & 104 & $38.0 \dagger$ & 1280 & 80 & $38.0 \dagger$ & 1304 \\
\hline Zargar et al (2008) [32] & India & Rural & 3024 & $20-40$ & 30.8 & 25 & - & - & - & - & - & - \\
\hline \multirow[t]{2}{*}{ Sadikot et al (2004) [33] } & \multirow[t]{2}{*}{ India } & Urban & 10617 & $\geq 25$ & 44.8 & 59 & 56 & 44.5 & 5379 & 58 & 45.1 & 5238 \\
\hline & & Rural & 7746 & $\geq 25$ & 44.2 & 25 & 25 & 44.1 & 3629 & 25 & 44.3 & 4117 \\
\hline Ramachandran et al (2001) [34] & India & Urban & 11216 & $\geq 20$ & 42.7 & 139 & 138 & 42.4 & 5288 & 140 & 43.0 & 5928 \\
\hline Hadaegh et al (2008) [35] & Iran & Urban & 9489 & $\geq 20$ & 43.5 & 142 & 141 & 44.8 & 4006 & 143 & 42.4 & 5483 \\
\hline Sadeghi et al (2007) [36] & Iran & Both & 12514 & $\geq 19$ & 39 & 63 & 54 & $39 \dagger$ & 6123 & 71 & $39 \dagger$ & 6391 \\
\hline Shrestha et al (2006) [37] & Nepal & Urban & 1012 & $\geq 40$ & 54.7 & 191 & 246 & $54.9 \ddagger$ & 423 & 151 & 54.5 丰 & 589 \\
\hline Katulanda et al (2008) [38] & Sri Lanka & Both & 4388 & $\geq 18$ & 46.1 & 126 & 98 & 46.3 & 1720 & 109 & 46 & 2668 \\
\hline
\end{tabular}

FPG - fasting plasma glucose, OGTT - oral glucose tolerance test

* Estimated mean age based on hypothetical maximum age of 80 .

$\uparrow$ Sex-specific mean ages not provided, overall mean age used for both sexes.

†stimated mean age based on age-group breakdowns.

Table 9. Individual study prevalence data: FPG-only studies

\begin{tabular}{|c|c|c|c|c|c|c|c|c|c|c|c|c|}
\hline Authors & Countity & $\begin{array}{l}\text { URBAN/ } \\
\text { RURAL }\end{array}$ & $\begin{array}{l}\text { Study } \\
\text { SAMPIF } \\
\text { SIZE }\end{array}$ & $\begin{array}{l}\text { AGE } \\
\text { RANGE OF } \\
\text { PARTICI- } \\
\text { PANTS }\end{array}$ & $\begin{array}{l}\text { MEAN } \\
\text { AGE }\end{array}$ & $\begin{array}{l}\text { OVERALL } \\
\text { DIABEITS } \\
\text { PREVAIFNCE } \\
\text { (11000 } \\
\text { POPULAIION) }\end{array}$ & $\begin{array}{l}\text { MALIE } \\
\text { DIABEETES } \\
\text { PREVALENCE } \\
\text { (1000 } \\
\text { MEN) }\end{array}$ & Mean age & $\begin{array}{l}\text { SizE OF } \\
\text { GROUP }\end{array}$ & $\begin{array}{l}\text { FeMALIF } \\
\text { DIABETES } \\
\text { PREVALENCE } \\
\text { (1000 } \\
\text { WOMEN) }\end{array}$ & $\begin{array}{l}\text { MeAN } \\
\text { AGE }\end{array}$ & $\begin{array}{l}\text { SIzE OF } \\
\text { GROUP }\end{array}$ \\
\hline Rahman et al (2007) [39] & Bangladesh & Rural & 975 & $\geq 20$ & 38.9 & 85 & 94 & 41.7 & 360 & 80 & 37.3 & 615 \\
\hline \multirow[t]{2}{*}{ Hussain et al (2005) [40] } & Bangladesh & Rural & 4757 & $\geq 20$ & 37.5 & 23 & 19 & $39.7 \dagger$ & 2030 & 25 & $35.8^{*}$ & 2720 \\
\hline & & Urban & 1555 & $\geq 20$ & 33.5 & 81 & 77 & $35.9 *$ & 731 & 85 & $31.4^{*}$ & 824 \\
\hline \multirow[t]{2}{*}{ Pandey et al (2013) [41] } & India & Rural & 2616 & $35-70$ & 46.7 & 43 & - & - & - & - & - & - \\
\hline & & Urban & 2008 & $35-70$ & 48.4 & 151 & - & - & - & - & - & - \\
\hline Vaz et al (2011) [42] & India & Rural & 1266 & $\geq 20$ & 39 & 103 & 84 & $39 \dagger$ & 609 & 120 & $39 \dagger$ & 657 \\
\hline Rao et al (2010) [43] & India & Rural & 1239 & $\geq 30$ & 51.3 & 160 & 188 & 50 & 434 & 144 & 52 & 805 \\
\hline Vijayakumar et al (2009) [44] & India & Rural & 1645 & $\geq 18$ & 47.2 & 146 & 165 & 48.2 & 624 & 135 & 46.2 & 1021 \\
\hline Namperumalsamy et al (2009) [45] & India & Both & 25969 & $\geq 30$ & 47 & 108 & - & - & - & - & - & - \\
\hline Chow et al (2006) [46] & India & Rural & 4538 & $\geq 30$ & 46.8 & 132 & - & - & - & - & - & - \\
\hline Gupta et al (2003) [47] & India & Both & 1091 & $\geq 20$ & 43.9 & 123 & 132 & 43.3 & 532 & 115 & 44.4 & 559 \\
\hline Misra et al (2001) [48] & India & Urban & 532 & $\geq 18$ & 35.4 & 103 & 112 & 37.8 & 170 & 99 & 34.3 & 362 \\
\hline Esteghamati et al (2009) [49] & Iran & Both & 3397 & $25-64$ & 44.2 & 87 & - & - & - & - & - & - \\
\hline Azimi-Nezhad et al (2008) [50] & Iran & Both & 3438 & $20-64$ & 48.5 & 55 & - & - & - & - & - & - \\
\hline Sharma et al (2011) [51] & Nepal & Both & 14008 & $\geq 20$ & 41.4 & 63 & 81 & $41.4 \dagger$ & 5326 & 53 & $41.4 \dagger$ & 8682 \\
\hline Paudyal et al (2008) [52] & Nepal & Rural & 1475 & $\geq 40$ & 54.7 & 41 & - & - & - & - & - & - \\
\hline Basit et al (2011) [53] & Pakistan & Rural & 1264 & $\geq 25$ & 42.3 & 142 & 165 & 43.5 & 424 & 131 & 41.7 & 840 \\
\hline Zafar et al (2011) [54] & Pakistan & Urban & 1091 & $12-80$ & 36 & 131 & 154 & $36 \dagger$ & 293 & 123 & $36 \dagger$ & 798 \\
\hline Mahar et al (2010) [55] & Pakistan & Urban & 19211 & $30-90$ & 42 & 87 & - & - & - & - & - & - \\
\hline Basit et al (2002) [56] & Pakistan & Rural & 2032 & $\geq 25$ & 38.9 & 72 & 119 & 40.4 & 670 & 49 & 38.1 & 1362 \\
\hline Pinidiyapathirage et al (2013) [57] & Sri Lanka & Urban & 2986 & $35-64$ & 52.3 & 247 & 233 & $52.2 \dagger$ & 1349 & 259 & $52.3 \dagger$ & 1637 \\
\hline \multirow{4}{*}{$\begin{array}{l}\text { Wijewardene et al (2005) [58] } \\
\text { Sri Lanka }\end{array}$} & & Urban & 4301 & $30-65$ & 46.8 & 175 & 183 & 46.4 & 1891 & 168 & 47.2 & 2410 \\
\hline & & Rural & 571 & $30-65$ & 44.6 & 175 & 73 & 44.7 & 275 & 67 & 44.6 & 297 \\
\hline & & Rural & 331 & $30-65$ & 45.9 & 48 & 50 & 45.4 & 139 & 47 & 46.3 & 192 \\
\hline & & Rural & 844 & $30-65$ & 45.7 & 71 & 70 & 45.6 & 387 & 72 & 45.8 & 457 \\
\hline
\end{tabular}

FPG - fasting plasma glucose

* Estimated mean age based on age-group breakdowns.

$\uparrow$ Sex-specific mean ages not provided, overall mean age used for both sexes. 
Table 10. Individual study prevalence data: OGTT-only studies

\begin{tabular}{|c|c|c|c|c|c|c|c|c|c|c|c|c|}
\hline Authors & Country & $\begin{array}{l}\text { Urban/ } \\
\text { Rural }\end{array}$ & $\begin{array}{l}\text { StudY } \\
\text { SAMPIE } \\
\text { SIZE }\end{array}$ & $\begin{array}{l}\text { Age RANGe } \\
\text { Of PARTICl- } \\
\text { PANTS }\end{array}$ & $\begin{array}{l}\text { Mean } \\
\text { Age }\end{array}$ & $\begin{array}{l}\text { Overall diabetes } \\
\text { Prevalence (V1000 } \\
\text { POPULATION) }\end{array}$ & $\begin{array}{l}\text { MaLE DIABETES } \\
\text { PREVALENGE } \\
\text { (11000 MEN) }\end{array}$ & $\begin{array}{l}\text { Mean } \\
\text { AGE }\end{array}$ & $\begin{array}{l}\text { SIzE Of } \\
\text { GROUP }\end{array}$ & $\begin{array}{l}\text { FEMALE DIABETES } \\
\text { PREVALENGE } \\
\text { (/1000 WOMEN) }\end{array}$ & $\begin{array}{l}\text { MeAN } \\
\text { AGE }\end{array}$ & $\begin{array}{l}\text { SizE of } \\
\text { GRoup }\end{array}$ \\
\hline Boddula et al (2008) [59] & India & Urban & 1112 & $\geq 30$ & $55^{*}$ & 246 & 284 & $55^{*}$ & 557 & 207 & $55^{*}$ & 555 \\
\hline Ramachandran et al (2004) [60] & India & Rural & 1213 & $\geq 20$ & 41 & 63 & 74 & 41.8 & 497 & 56 & 40.5 & 716 \\
\hline \multirow[t]{2}{*}{ Ramachandran et al (1994) [61] } & \multirow[t]{2}{*}{ India } & Urban & 873 & $\geq 60$ & $70 *$ & 237 & - & - & - & - & - & - \\
\hline & & Rural & 588 & $\geq 60$ & $70^{*}$ & 99 & - & - & - & - & - & - \\
\hline \multirow[t]{2}{*}{ Ramachandran et al (1992) [62] } & \multirow[t]{2}{*}{ India } & Urban & 900 & $\geq 20$ & 38 & 82 & 103 & 40 & 457 & 61 & 37 & 443 \\
\hline & & Rural & 1038 & $\geq 20$ & 41 & 24 & 27 & 41 & 520 & 21 & 41 & 518 \\
\hline
\end{tabular}

OGTT - oral glucose tolerance test

* Estimated mean age based on hypothetical maximum age of 80 .

Table 11. Overall prevalence estimates for 2005

\begin{tabular}{|c|c|c|c|c|c|}
\hline $\begin{array}{l}\text { Age } \\
\text { RANGE }\end{array}$ & $\begin{array}{l}\text { MEAN } \\
\text { AGE } \\
\text { (YEARS) }\end{array}$ & $\begin{array}{l}\text { PAEI/AIENGE / } \\
1000 \text { POPULATION } \\
\left(\mathrm{y}=0.0368 \mathrm{x}^{2.039}\right)\end{array}$ & $\begin{array}{l}2005 \\
\text { UNPD } \\
\text { POPULATION } \\
\text { ESTIMATES } \\
(\times 1000)\end{array}$ & $\begin{array}{l}\text { CALCULATED } \\
2005 \\
\text { PREVALENCE } \\
\text { ESTIMATES } \\
(\times 1000)\end{array}$ & $\begin{array}{l}\text { Proportion } \\
\text { OF BURdeN } \\
\text { BY AGE } \\
\text { GROUP }(\%)\end{array}$ \\
\hline $20-24$ & 22 & 20.09 & 152031 & 3055 & 4.64 \\
\hline $25-29$ & 27 & 30.51 & 134001 & 4088 & 6.21 \\
\hline $30-34$ & 32 & 43.14 & 115491 & 4982 & 7.57 \\
\hline $35-39$ & 37 & 58.00 & 102984 & 5973 & 9.07 \\
\hline $40-44$ & 42 & 75.10 & 89614 & 6730 & 10.22 \\
\hline $45-49$ & 47 & 94.46 & 76802 & 7255 & 11.02 \\
\hline $50-54$ & 52 & 116.09 & 64131 & 7445 & 11.31 \\
\hline $55-59$ & 57 & 139.98 & 47010 & 6581 & 9.98 \\
\hline $60-64$ & 62 & 166.16 & 37303 & 6198 & 9.41 \\
\hline $65-69$ & 67 & 194.63 & 29394 & 5721 & 8.69 \\
\hline $70-74$ & 72 & 225.40 & 20538 & 4629 & 7.03 \\
\hline $75-79$ & 77 & 258.47 & 12348 & 3191 & 4.85 \\
\hline & & Total: & 881647 & 65848 & 100.00 \\
\hline
\end{tabular}

UNPD- United Nations Population Division

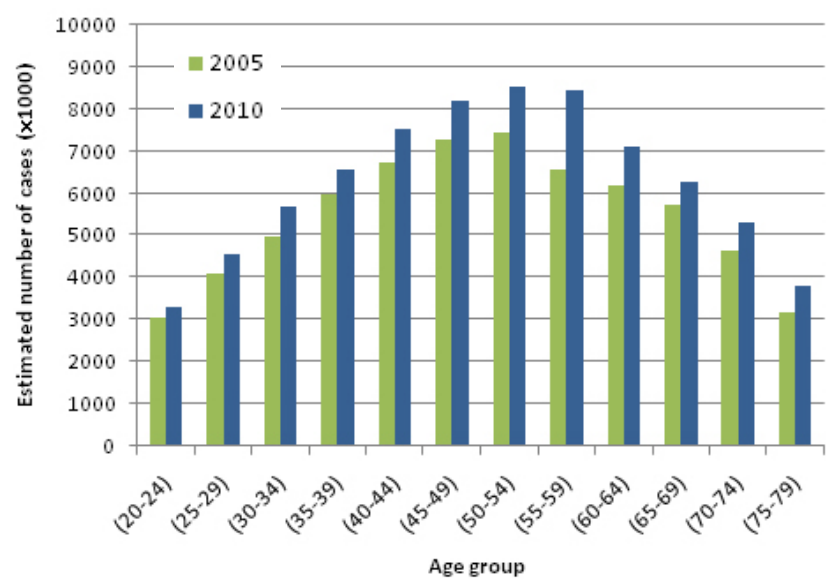

Figure 4. Estimated numbers of type 2 diabetes cases in Southern Asia (in thousands) by age group in 2005 and 2010.

2010 than for 2005: 7.60\% compared to $7.47 \%$. Figure 5 and Table 13 illustrate the relationship between diabetes prevalence and age for males (all diagnostic methods). Based on the trend line equations, male prevalence is slightly higher than female prevalence at all ages. At the extreme, estimated male prevalence for the 70-79 age group is 336.08 per 1000 , whereas female prevalence is 297.57 per 1000. Figure 6 and Table 14 illustrate female age-specific prevalence for all diagnostic methods. Sex-and age-specific prevalence data are presented in Supplementary Online Document.
Table 12. Overall prevalence estimates for 2010

\begin{tabular}{|c|c|c|c|c|c|}
\hline $\begin{array}{l}\text { AgE } \\
\text { RANGE }\end{array}$ & $\begin{array}{l}\text { MeAN } \\
\text { AGE } \\
\text { (YeArs) }\end{array}$ & $\begin{array}{l}\text { PREVAILNCE / } \\
1000 \text { POPULATION } \\
\left(\mathrm{r}=0.0368 \mathrm{x}^{2.039}\right)\end{array}$ & $\begin{array}{l}2010 \\
\text { UNPD } \\
\text { POPULATION } \\
\text { ESTIMAIES } \\
(\times 1000)\end{array}$ & $\begin{array}{l}\text { CalCulated } \\
2010 \text { PREVA- } \\
\text { LENCE ESTIMATES } \\
(\times 1000)\end{array}$ & $\begin{array}{l}\text { PROPORTION } \\
\text { OF BURDEN BY } \\
\text { AGE GROUP } \\
(\%)\end{array}$ \\
\hline $20-24$ & 22 & 20.09 & 163363 & 3282 & 4.36 \\
\hline $25-29$ & 27 & 30.51 & 149024 & 4546 & 6.04 \\
\hline $30-34$ & 32 & 43.14 & 131390 & 5668 & 7.53 \\
\hline $35-39$ & 37 & 58.00 & 112937 & 6550 & 8.70 \\
\hline $40-44$ & 42 & 75.10 & 100399 & 7540 & 10.01 \\
\hline $45-49$ & 47 & 94.46 & 86776 & 8197 & 10.89 \\
\hline $50-54$ & 52 & 116.09 & 73608 & 8545 & 11.35 \\
\hline $55-59$ & 57 & 139.98 & 60318 & 8444 & 11.21 \\
\hline $60-64$ & 62 & 166.16 & 42750 & 7103 & 9.43 \\
\hline $65-69$ & 67 & 194.63 & 32320 & 6291 & 8.35 \\
\hline $70-74$ & 72 & 225.40 & 23627 & 5325 & 7.07 \\
\hline $75-79$ & 77 & 258.47 & 14742 & 3810 & 5.06 \\
\hline & & Total: & 991254 & 75302 & 100.00 \\
\hline
\end{tabular}

UNPD- United Nations Population Division

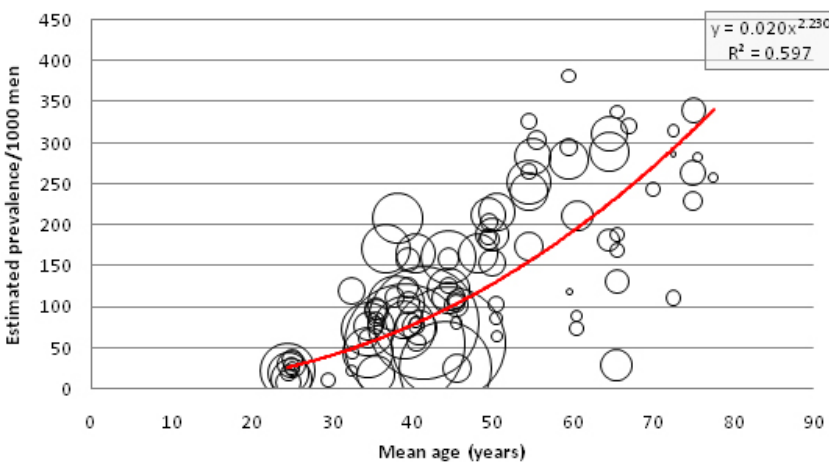

Figure 5. Relationship between crude prevalence of type 2 diabetes and age in Southern Asia in male examinees.

Table 13. Male prevalence estimates for 2005

\begin{tabular}{|c|c|c|c|c|c|}
\hline Age range & $\begin{array}{l}\text { M/EAN } \\
\text { AGE } \\
\text { (VFARS) }\end{array}$ & $\begin{array}{l}\text { PREVIA- } \\
\text { LENCE/1000 MEN } \\
\left(\mathrm{r}=0.0208 \mathrm{x}^{2.2308}\right)\end{array}$ & $\begin{array}{l}2005 \\
\text { UNPD MALE } \\
\text { POPULATION } \\
\text { ESTIMATES } \\
(\times 1000)\end{array}$ & $\begin{array}{l}\text { CalCULATEd } \\
2005 \text { MALE } \\
\text { PREVALENCE } \\
\text { ESTIMATES } \\
(\times 1000)\end{array}$ & $\begin{array}{l}\text { PROPOR- } \\
\text { TION OF } \\
\text { BURDEN BY } \\
\text { AGE GROUP } \\
(\%)\end{array}$ \\
\hline $20-24$ & 22 & 20.55 & 78574 & 1614 & 4.07 \\
\hline $25-29$ & 27 & 32.45 & 69157 & 2244 & 5.66 \\
\hline $30-34$ & 32 & 47.40 & 59699 & 2830 & 7.14 \\
\hline $35-39$ & 37 & 65.53 & 53302 & 3493 & 8.81 \\
\hline $40-44$ & 42 & 86.94 & 46349 & 4029 & 10.17 \\
\hline $45-49$ & 47 & 111.73 & 39741 & 4440 & 11.21 \\
\hline $50-54$ & 52 & 140.00 & 32984 & 4618 & 11.65 \\
\hline $55-59$ & 57 & 171.82 & 23669 & 4067 & 10.26 \\
\hline $60-64$ & 62 & 207.27 & 18546 & 3844 & 9.70 \\
\hline $65-69$ & 67 & 246.42 & 14386 & 3545 & 8.95 \\
\hline $70-74$ & 72 & 289.34 & 9985 & 2889 & 7.29 \\
\hline $75-79$ & 77 & 336.08 & 5979 & 2009 & 5.07 \\
\hline & & Total: & 452371 & 39622 & 100.00 \\
\hline & & & $\frac{2005 \text { male }}{2005 \text { corre }}$ & $\begin{array}{l}\text { prevalence } \\
\text { ted prevale }\end{array}$ & $\frac{8.76 \%}{\text { ce: } 7.72 \%}$ \\
\hline
\end{tabular}

UNPD- United Nations Population Division 


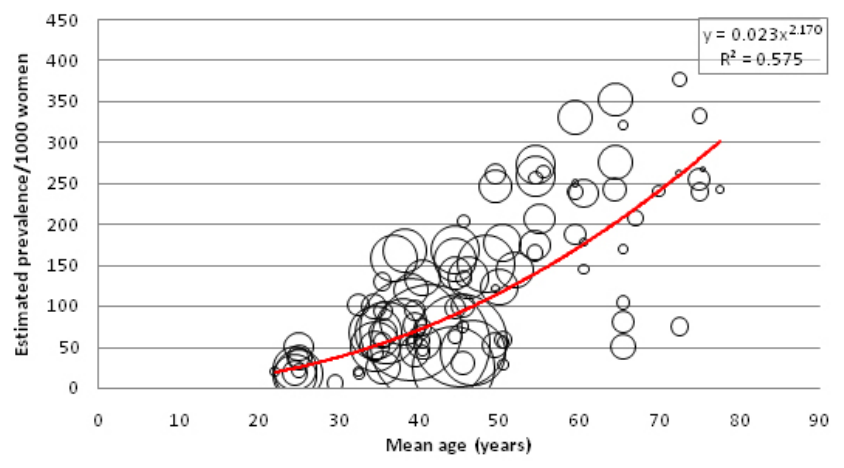

Figure 6. Relationship between crude prevalence of type 2 diabetes and age in Southern Asia in female examinees.

Table 14. Female prevalence estimates for 2005

\begin{tabular}{|c|c|c|c|c|c|}
\hline $\begin{array}{l}\text { AgE } \\
\text { RANGE }\end{array}$ & $\begin{array}{l}\text { MeAM } \\
\text { AGE } \\
\text { (YEARS) }\end{array}$ & $\begin{array}{l}\text { Prevalence/1000 } \\
\text { wOMEN } \\
\left(y=0.0239 x^{2.1708}\right)\end{array}$ & $\begin{array}{l}2005 \text { UNPD } \\
\text { FEMALE } \\
\text { POPULATION } \\
\text { ESTIMATES } \\
(\times 1000)\end{array}$ & $\begin{array}{l}\text { CalCULATED } \\
2005 \text { FEMALE } \\
\text { PREVALENCE } \\
\text { ESTIMATES } \\
\text { (1000) }\end{array}$ & $\begin{array}{l}\text { Proportion } \\
\text { OF BURDEN } \\
\text { BY AGE } \\
\text { GROUP }\end{array}$ \\
\hline $20-24$ & 22 & 19.61 & 73457 & 1441 & $4.10 \%$ \\
\hline $25-29$ & 27 & 30.59 & 64844 & 1984 & $5.65 \%$ \\
\hline $30-34$ & 32 & 44.24 & 55792 & 2468 & $7.03 \%$ \\
\hline $35-39$ & 37 & 60.62 & 49682 & 3012 & $8.58 \%$ \\
\hline $40-44$ & 42 & 79.83 & 43265 & 3454 & $9.84 \%$ \\
\hline $45-49$ & 47 & 101.90 & 37061 & 3777 & $10.76 \%$ \\
\hline $50-54$ & 52 & 126.91 & 31147 & 3953 & $11.26 \%$ \\
\hline $55-59$ & 57 & 154.90 & 23341 & 3616 & $10.30 \%$ \\
\hline $60-64$ & 62 & 185.92 & 18757 & 3487 & $9.93 \%$ \\
\hline $65-69$ & 67 & 220.01 & 15008 & 3302 & $9.41 \%$ \\
\hline $70-74$ & 72 & 257.21 & 10553 & 2714 & $7.73 \%$ \\
\hline $75-79$ & 77 & 297.57 & 6368 & 1895 & $5.40 \%$ \\
\hline & & Total: & 429275 & 35102 & $100.00 \%$ \\
\hline & & & \multicolumn{3}{|c|}{$\begin{array}{l}2005 \text { female prevalence: } 8.18 \% \\
2005 \text { corrected prevalence: } 7.20 \%\end{array}$} \\
\hline
\end{tabular}

UNPD- United Nations Population Division

The crude population prevalence estimate for males in 2005 is $8.76 \%$ (39622000 cases), and for females is $8.18 \%$ (35 102000 cases) $(P<0.001)$. These do not total the combined prevalence estimate of 65848000 cases $(7.47 \%)$ since several studies did not provide enough data to calculate male and female age-specific prevalence, only enough to calculate combined sexes age-specific prevalence. To account for this incomplete data, the combined prevalence estimate was used as an envelope and a correction factor of 0.881 was applied to male and female prevalence estimates. Resultantly, the adjusted 2005 population prevalence estimates are $7.72 \%$ for males and $7.20 \%$ for females. Figure 7 illustrates a comparison between corrected male and female prevalence estimates at 5-year age intervals.

\section{Urban and rural residency}

32 out of the 39 selected studies (50 out of 57 cohorts) specifically defined their study population as residing in an urban area or a rural area. Incidentally, out of the reporting studies, 25 cohorts were specified as urban and 25 as rural. Figure 8 illustrates diabetes prevalence against age in ur-

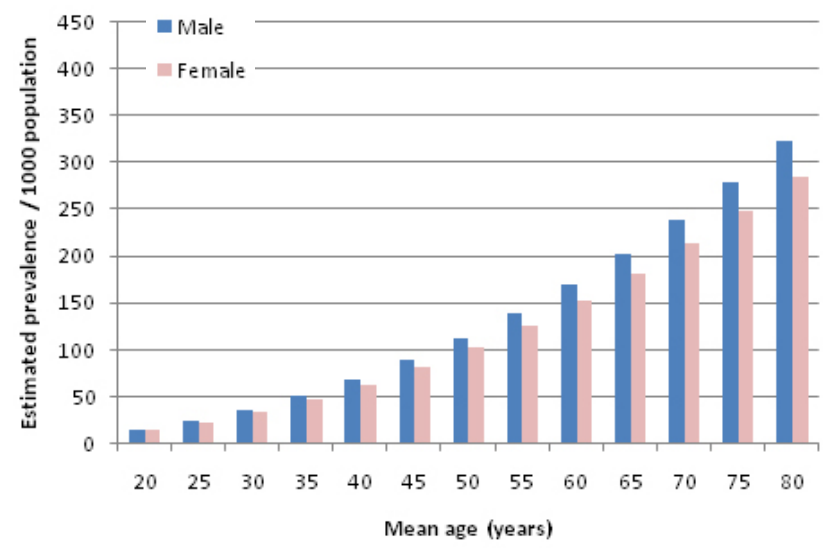

Figure 7. Male and female prevalence of type 2 diabetes in Southern Asia in 2005, adjusted to an "envelope" defined by all studies with prevalence data.

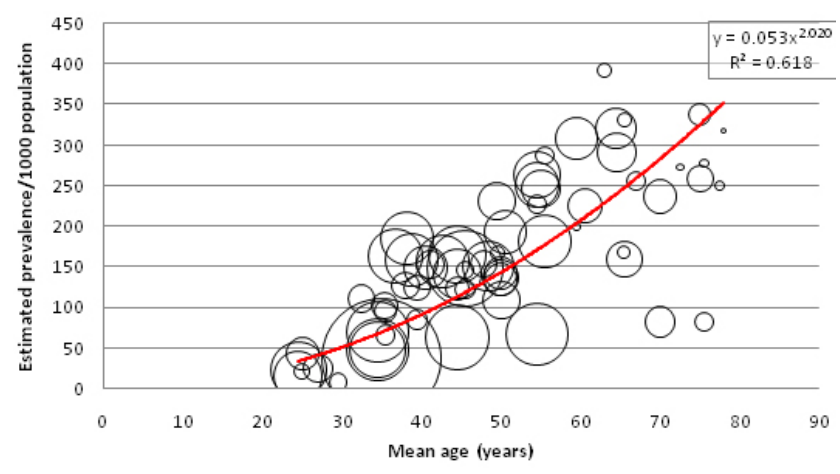

Figure 8. Crude prevalence of type 2 diabetes in Southern Asia in urban regions and its relationship with age.

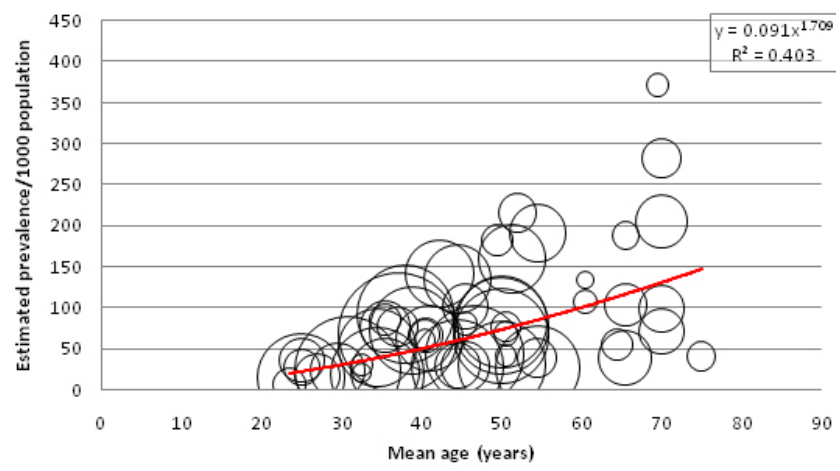

Figure 9. Crude prevalence of type 2 diabetes in Southern Asia in rural regions and its relationship with age.

ban cohorts (both sexes, all diagnostic methods), and Figure 9 does likewise for rural cohorts. Table 15 and Figure 10 highlight the prevalence differences observed between urban and rural cohorts. Figure 11 compares prevalence of diabetes in urban males with prevalence in rural males. Figure 12 compares prevalence of urban and rural females. In both cases urban residency is associated with signifi- 
Table 15. Urban and rural overall prevalence comparison $(P<0.001)$

\begin{tabular}{|c|c|c|}
\hline Age (Years) & $\begin{array}{l}\text { EstiMATED URBAN PREVALENGE/1000 } \\
\text { POPULATION }\left(\mathrm{Y}=0.053 \mathrm{x}^{2.0206}\right)\end{array}$ & $\begin{array}{l}\text { EstiMATED RURAL PREVALENGE/1000 } \\
\left.\text { POPULATION ( } \mathrm{y}=0.0917 \mathrm{x}^{1.7091}\right)\end{array}$ \\
\hline 20 & 22.55 & 15.34 \\
\hline 25 & 35.40 & 22.47 \\
\hline 30 & 51.16 & 30.68 \\
\hline 35 & 69.86 & 39.93 \\
\hline 40 & 91.50 & 50.17 \\
\hline 45 & 116.08 & 61.36 \\
\hline 50 & 143.62 & 73.46 \\
\hline 55 & 174.12 & 86.46 \\
\hline 60 & 207.59 & 100.32 \\
\hline 65 & 244.03 & 115.03 \\
\hline 70 & 283.45 & 130.56 \\
\hline 75 & 325.86 & 146.90 \\
\hline
\end{tabular}

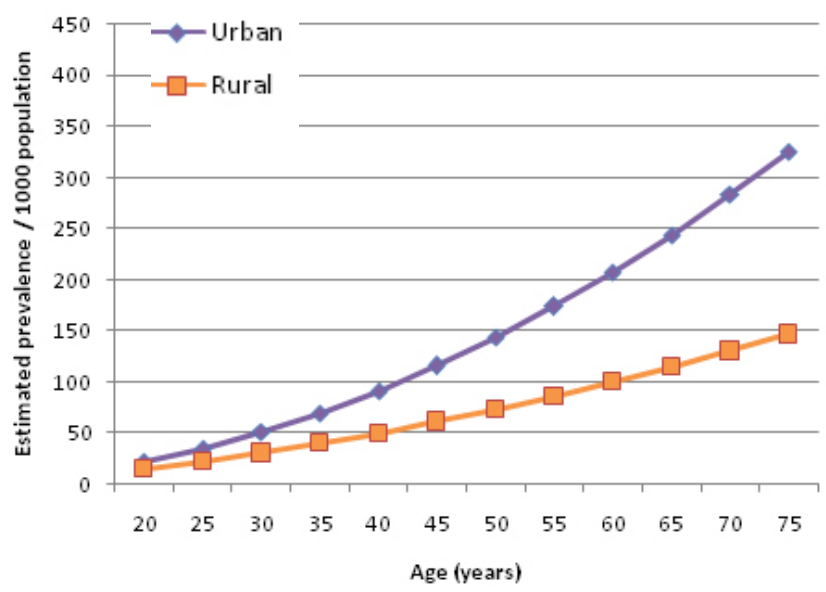

Figure 10. Comparison of the prevalence of type 2 diabetes in Southern Asia by age in urban and rural regions - both sexes included.

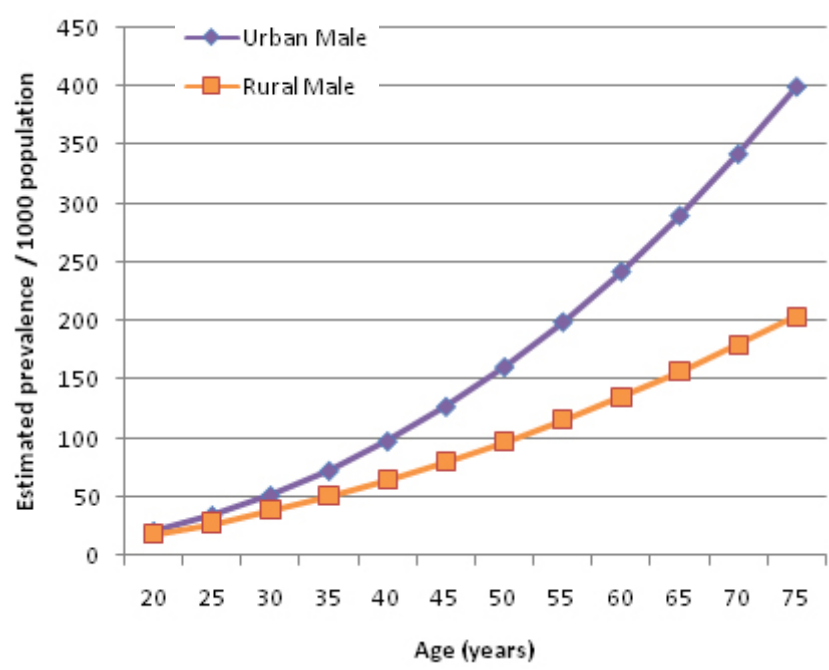

Figure 11. Comparison of the prevalence of type 2 diabetes in Southern Asia by age in urban and rural regions - men only.

cantly higher diabetes prevalence $(P<0.001)$. Figures 13 and 14 show the estimates for both sexes by residency. Individual bubble graphs for residency are presented in Online Supplementary Document.

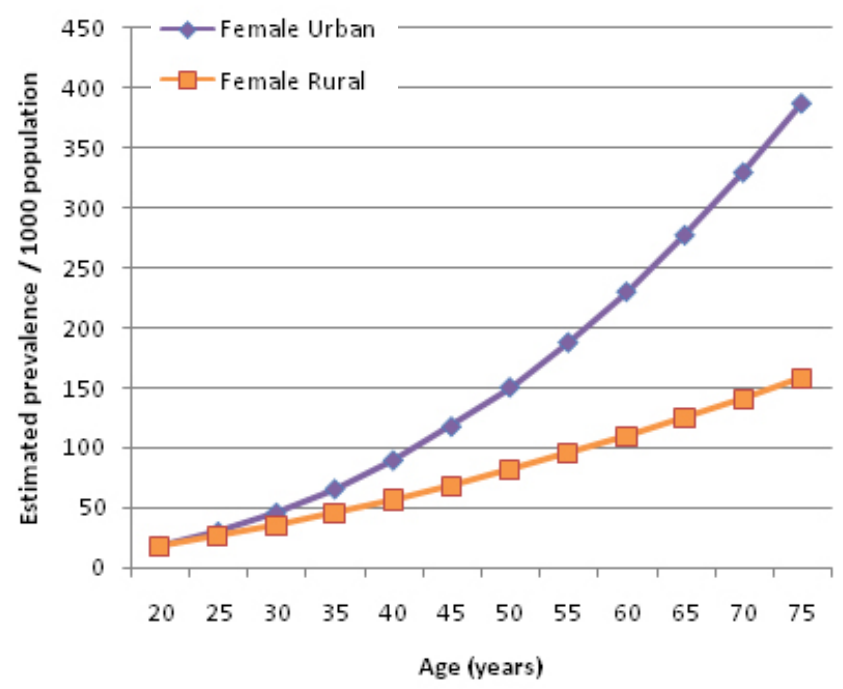

Figure 12. Comparison of the prevalence of type 2 diabetes in Southern Asia by age in urban and rural regions - women only.

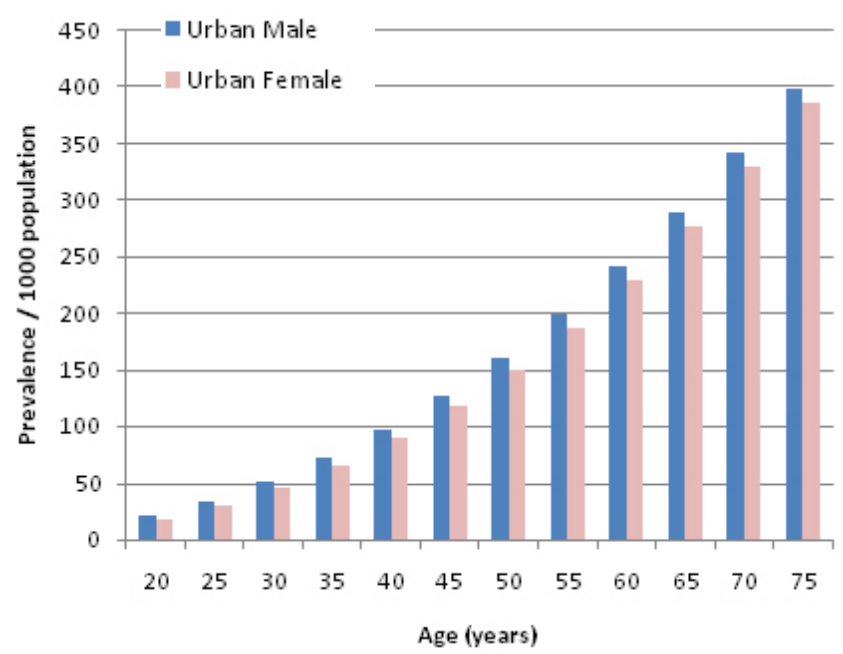

Figure 13. Male and female prevalence of type 2 diabetes in Southern Asia based on information from urban regions.

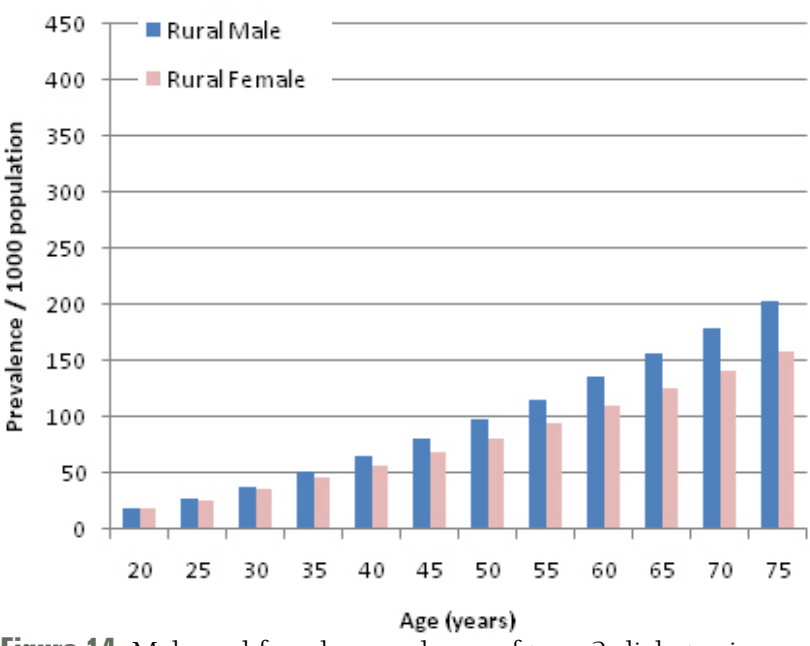

Figure 14. Male and female prevalence of type 2 diabetes in Southern Asia based on information from rural regions. 


\section{Method of diabetes diagnosis}

Figure 15 shows the relationship between age and diabetes prevalence when only considering studies that utilised both FPG and OGTT in diagnosing diabetes. Figure 16 shows the relationship for studies that used only FPG, and Figure 17 shows OGTT-only studies. 65 age-specific data points were used to calculate the FPG+OGTT trend line, 77 data points used to calculate the FPG-only trend line, but due to the small number of available OGTT studies only 9 age-specific data points were used in calculating the OGTT trend line. Figure 18 compares the estimated prevalence using each of the three diagnostic methods. The combined FPG plus OGTT studies resulted in a higher population prevalence estimate than FPG-only studies when applied to UNPD figures, as shown by Table 16 and Table 17. FPG plus OGTT studies result in a population prevalence of $7.75 \%$, while FPG-only studies result in a prevalence of $7.32 \%$. The small number of OGTT-only studies predicted a population prevalence of $6.95 \%$, as shown in Table 18.

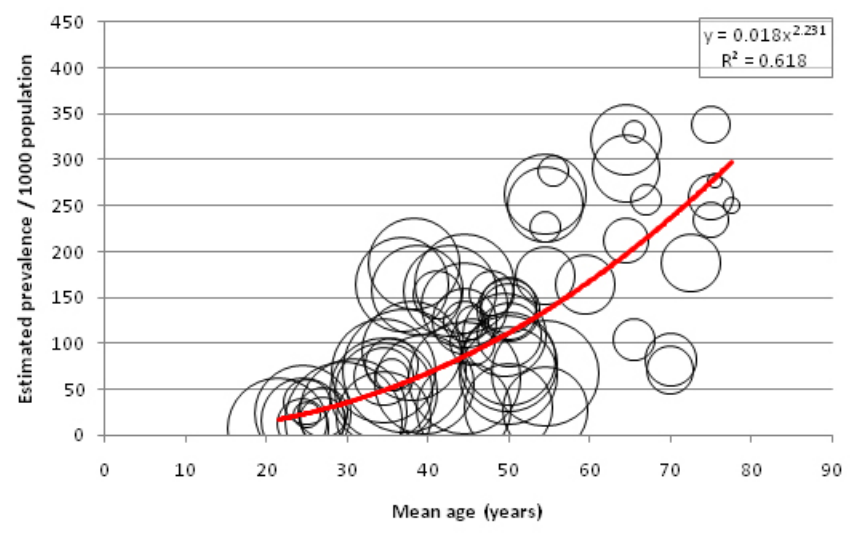

Figure 15. Relationship between crude prevalence of type 2 diabetes and age in Southern Asia based on the studies using FPG+OGTT in their case definition. FPG - fasting plasma glucose, OGTT - oral glucose tolerance test.

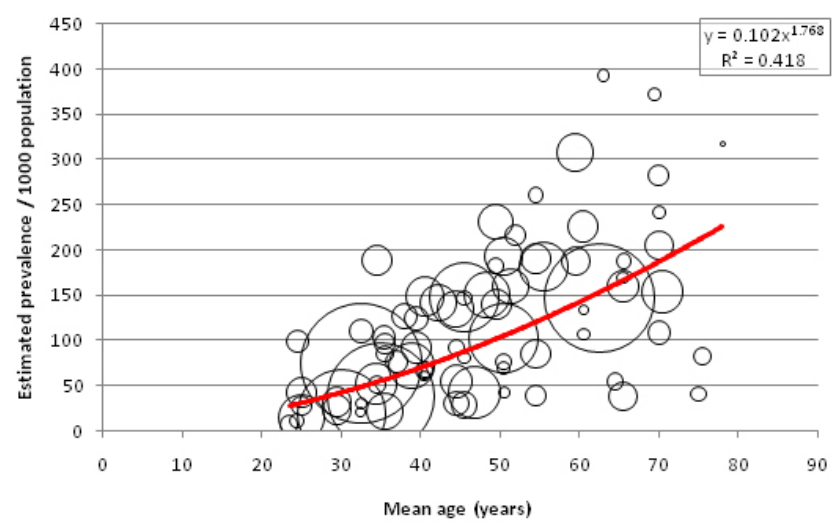

Figure 16. Relationship between crude prevalence of type 2 diabetes and age in Southern Asia based on the studies using FPG only in their case definition. FPG - fasting plasma glucose.
Table 16. Prevalence estimates for 2005 based on FPG+OGTT studies

\begin{tabular}{|c|c|c|c|c|c|}
\hline $\begin{array}{l}\text { Age } \\
\text { RANGE }\end{array}$ & $\begin{array}{l}\text { MeAN } \\
\text { AGE } \\
\text { (YEARS) }\end{array}$ & $\begin{array}{l}\text { Prevalence/1000 } \\
\text { Population } \\
\left(\mathrm{v}=0.0181 \mathrm{x}^{2.2319}\right)\end{array}$ & $\begin{array}{l}2005 \text { UNPD } \\
\text { POPULATION } \\
\text { ESTIMATES } \\
(\times 1000)\end{array}$ & $\begin{array}{l}\text { CALCU- } \\
\text { LATED 2005 } \\
\text { PREVALENCE } \\
\text { ESTIMATES } \\
\text { ( } \times 1000)\end{array}$ & $\begin{array}{l}\text { Proportion } \\
\text { OF BURDEN } \\
\text { BY AGE } \\
\text { GROUP (\%) }\end{array}$ \\
\hline $20-24$ & 22 & 17.94 & 152031 & 2727 & 3.99 \\
\hline $25-29$ & 27 & 28.34 & 134001 & 3797 & 5.56 \\
\hline $30-34$ & 32 & 41.40 & 115491 & 4782 & 7.00 \\
\hline $35-39$ & 37 & 57.25 & 102984 & 5895 & 8.63 \\
\hline $40-44$ & 42 & 75.96 & 89614 & 6807 & 9.96 \\
\hline $45-49$ & 47 & 97.64 & 76802 & 7499 & 10.97 \\
\hline $50-54$ & 52 & 122.36 & 64131 & 7847 & 11.48 \\
\hline $55-59$ & 57 & 150.18 & 47010 & 7060 & 10.33 \\
\hline $60-64$ & 62 & 181.18 & 37303 & 6759 & 9.89 \\
\hline $65-69$ & 67 & 215.42 & 29394 & 6332 & 9.27 \\
\hline $70-74$ & 72 & 252.96 & 20538 & 5195 & 7.60 \\
\hline $75-79$ & 77 & 293.86 & 12348 & 3629 & 5.31 \\
\hline & & Total: & 881647 & 68330 & 100.00 \\
\hline & & & \multicolumn{3}{|c|}{$\begin{array}{l}\text { FPG+OGTT population } \\
\text { prevalence: } 7.75 \%\end{array}$} \\
\hline & & & \multicolumn{3}{|c|}{$\begin{array}{l}\text { Overall population prevalence } \\
\text { (2005): } 7.47 \%\end{array}$} \\
\hline
\end{tabular}

FPG - fasting plasma glucose, OGTT - oral glucose tolerance test, UNPDUnited Nations Population Division

Table 17. Prevalence estimates for 2005 based on FPG-only studies

\begin{tabular}{|c|c|c|c|c|c|}
\hline $\begin{array}{l}\text { Age } \\
\text { RAMGE }\end{array}$ & $\begin{array}{l}\text { MEAN } \\
\text { AGE } \\
\text { (YEARS) }\end{array}$ & $\begin{array}{l}\text { Prevaliance/1000 } \\
\text { POPULATION } \\
\left(\mathrm{y}=0.1021 \mathrm{x}^{1.7684}\right)\end{array}$ & $\begin{array}{l}2005 \text { UNPD } \\
\text { POPULATION } \\
\text { ESTIMATES } \\
(\times 1000)\end{array}$ & $\begin{array}{l}\text { CalCU- } \\
\text { LATED } 2005 \\
\text { PREVALENCE } \\
\text { ESTIMATES } \\
(\times 1000)\end{array}$ & $\begin{array}{l}\text { PropoRtION OF } \\
\text { BURDEN BY AGE } \\
\text { GROUP }\end{array}$ \\
\hline $20-24$ & 22 & 24.15 & 152031 & 3672 & $5.69 \%$ \\
\hline $25-29$ & 27 & 34.69 & 134001 & 4649 & $7.20 \%$ \\
\hline $30-34$ & 32 & 46.85 & 115491 & 5411 & $8.38 \%$ \\
\hline $35-39$ & 37 & 60.57 & 102984 & 6237 & $9.66 \%$ \\
\hline $40-44$ & 42 & 75.78 & 89614 & 6791 & $10.52 \%$ \\
\hline $45-49$ & 47 & 92.46 & 76802 & 7101 & $11.00 \%$ \\
\hline $50-54$ & 52 & 110.56 & 64131 & 7090 & $10.98 \%$ \\
\hline $55-59$ & 57 & 130.05 & 47010 & 6114 & $9.47 \%$ \\
\hline $60-64$ & 62 & 150.90 & 37303 & 5629 & $8.72 \%$ \\
\hline $65-69$ & 67 & 173.08 & 29394 & 5088 & $7.88 \%$ \\
\hline $70-74$ & 72 & 196.58 & 20538 & 4037 & $6.25 \%$ \\
\hline $75-79$ & 77 & 221.36 & 12348 & 2733 & $4.23 \%$ \\
\hline & & Total: & 88647 & 64553 & $100.00 \%$ \\
\hline & & & \multicolumn{3}{|c|}{$\begin{array}{c}\text { FPG-only population prevalence: } \\
7.32 \%\end{array}$} \\
\hline & & & \multicolumn{3}{|c|}{$\begin{array}{l}\text { Overall population prevalence } \\
\text { (2005): } 7.47 \%\end{array}$} \\
\hline
\end{tabular}

FPG - fasting plasma glucose, UNPD- United Nations Population Division

Table 18. Prevalence estimates for 2005 based on OGTT-only studies

\begin{tabular}{|c|c|c|c|c|c|}
\hline $\begin{array}{l}\text { Age } \\
\text { RANGE }\end{array}$ & $\begin{array}{l}\text { Mean } \\
\text { AgE } \\
\text { (YeArs) }\end{array}$ & $\begin{array}{l}\text { Prevalenge/1000 } \\
\text { POPULATION } \\
\left(\mathrm{r}=0.033 \mathrm{x}^{2.1118}\right)\end{array}$ & $\begin{array}{l}2005 \text { UNPD } \\
\text { POPULATION ESTI- } \\
\text { MATES ( } \times 1000)\end{array}$ & $\begin{array}{l}\text { CALCU- } \\
\text { LATEd 2005 } \\
\text { PREVALENCE } \\
\text { ESTIMATES } \\
(\times 1000)\end{array}$ & $\begin{array}{l}\text { Propor- } \\
\text { TION OF } \\
\text { BURDEN BY } \\
\text { AGE GROUP }\end{array}$ \\
\hline $20-24$ & 22 & 8.52 & 152031 & 1295 & 2.11 \\
\hline $25-29$ & 27 & 15.74 & 134001 & 2109 & 3.44 \\
\hline $30-34$ & 32 & 26.21 & 115491 & 3026 & 4.94 \\
\hline $35-39$ & 37 & 40.51 & 102984 & 4172 & 6.81 \\
\hline $40-44$ & 42 & 59.25 & 89614 & 5309 & 8.66 \\
\hline $45-49$ & 47 & 83.03 & 76802 & 6377 & 10.41 \\
\hline $50-54$ & 52 & 112.44 & 64131 & 7211 & 11.77 \\
\hline $55-59$ & 57 & 148.09 & 47010 & 6962 & 11.36 \\
\hline $60-64$ & 62 & 190.58 & 37303 & 7109 & 11.60 \\
\hline $65-69$ & 67 & 240.51 & 29394 & 7070 & 11.54 \\
\hline $70-74$ & 72 & 298.47 & 20538 & 6130 & 10.00 \\
\hline $75-79$ & 77 & 365.07 & 12348 & 4508 & 7.36 \\
\hline & & Total: & 881,647 & 61278 & 100.00 \\
\hline & & & \multicolumn{3}{|c|}{$\begin{array}{c}\text { OGTT-only population prevalence: } \\
6.95 \%\end{array}$} \\
\hline & & & \multicolumn{3}{|c|}{$\begin{array}{c}\text { Overall population prevalence } \\
\text { (2005): } 7.47 \%\end{array}$} \\
\hline
\end{tabular}

OGTT - oral glucose tolerance test, UNPD-United Nations Population Division 


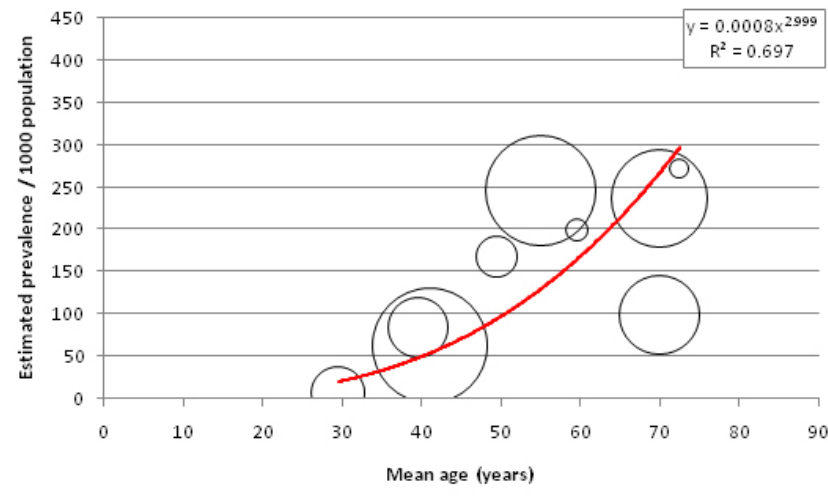

Figure 17. Relationship between crude prevalence of type 2 diabetes and age in Southern Asia based on the studies using OGTT only in their case definition. OGTT -oral glucose tolerance test.

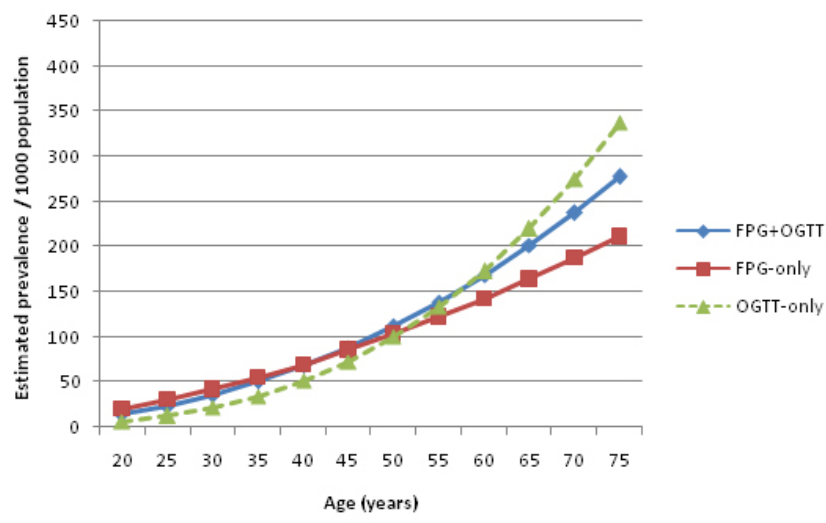

Figure 18. Comparison of the relationship between crude prevalence of type 2 diabetes and age in Southern Asia depending on the diagnostic methods used to establish case definition.

\section{DISCUSSION}

This study provides the most up-to-date transparent estimation of diabetes prevalence in the UN Southern Asia region, building upon previous studies looking at prevalence in comparable regions or in specific constituent countries $[21,22,63,64]$. It is also, to our knowledge, the first study to transparently estimate diabetes prevalence and trends in Southern Asia by synthesizing findings from numerous community-based studies in addition to broader national population studies.

A transparent systematic literature review of two online databases was carried out. Pre-defined inclusion and quality criteria narrowed down 39 studies from an initial 5653 results. Search terms were specified, and quality assessment criteria for selected studies were provided in Appendices. Enough studies were captured for estimation of overall diabetes prevalence, male and female prevalence, and urban and rural prevalence for each sex. Study cohort sites had a wide geographic distribution within the countries that were analysed, as shown in Figure 2. Additionally there was an even split of rural and urban studies, allowing for comparison of prevalence in both demographics. Data was not captured from all countries in the Southern Asia region the systematic literature review did not find any suitable studies from Afghanistan, Bhutan, or the Maldives. However, Table 5 shows that these three countries have the smallest adult populations in the region. For all other countries, including the three most populous countries of India, Pakistan, and Bangladesh, suitable numbers of geographically dispersed studies were identified. While this paper was able to adequately meet most of its set objectives, the small number of OGTT-only studies captured in the search meant that all recognised diagnostic methods could not be fully compared in terms of prevalence estimates, and so this objective was only partially met.

By applying this study's prevalence estimates to UNPD population Figures [23], the overall diabetes prevalence for the Southern Asia region was estimated to be $7.47 \%$ for 2005 , and $7.60 \%$ for 2010 . Although the reviewed studies were more representative of the year 2005, it is interesting to note the effects of an ageing population on diabetes prevalence. Estimates indicate that $25.0 \%$ of the regional population was aged 50 or older in 2010 , compared to $23.9 \%$ in 2005. In addition, UNPD projections for Southern Asia predict that total population and life expectancy at birth for both males and females will continue to rise in the region over the next 30 years [23]. The findings of this study suggest that as the population continues to age in the future, the overall burden of diabetes in Southern Asia will also continue to increase, and that concerted policy action is needed to facilitate the response to this increased burden.

It was found that diabetes prevalence was consistently higher for males than for females. The burden was highest in the 50-54 age group for both sexes, and within this age group there was a $9.35 \%$ difference between estimated male and female prevalence. Prevalence/1000 population continued to increase with age for both sexes but due to the population age structure the burden attributed to older age groups was progressively smaller after the ages of 50-54. After correction for missing data, the 2005 population prevalence estimate for males was $7.72 \%$, and for females was $7.20 \%$. This translates into an estimated 34915000 male and 30933000 female diabetics in Southern Asia in 2005.

This study found that urban residency was strongly associated with higher diabetes prevalence for both sexes. The observed difference is noteworthy - past the age of 55 the urban prevalence was estimated to be more than twice the rural prevalence. Although males had higher prevalence than females in both urban and rural settings, the difference was noticeably smaller in urban cohorts than rural cohorts: a $6.03 \%$ difference between urban males and females, but a $16.74 \%$ difference between their rural coun- 
terparts. Higher rates of diabetes among urban residents may be explained through increases in physical inactivity and consumption of high sugar and fat diets - both strong risk factors for diabetes - that have become synonymous with urban lifestyles. Mohan suggested that diabetes rates in India are quickly escalating because of the rapid urbanisation that is sweeping the country [17]. Conversely, rural prevalence remains lower because of limited exposure to these risk factors and maintenance of traditional physically vigorous rural lifestyles.

Sufficient numbers of FPG-only and FPG+OGTT combined studies were identified to allow comparison between these two diagnostic methods. The small number of OGTT-only studies also provided interesting trends. Overall the differences between these methods appeared to be minimal. The higher prevalence estimates of FPG+OGTT compared to FPG-only were to be expected since the former used an additional diagnostic method. FPG-only studies estimated higher diabetes prevalence at younger ages $(<52)$ and lower prevalence at older ages (>52) compared to OGTT-only. Combined FPG+OGTT prevalence estimates lay in between the FPG and OGTT estimates at both ends of the age spectrum. In addition, FPG-only studies estimated the highest burden proportion to be at a younger age - in the 45-49 age group - followed by a small decrease in the 50-54 age group. FPG+OGTT studies and the limited number of OGTT-only studies found highest burden proportion in the 50-54 age group. These findings suggest that FPG may have greater sensitivity at younger ages, while OGTT may be more sensitive to diabetes in older age.

This study's prevalence estimates were primarily based on trend line equations obtained by plotting study size, mean age and prevalence estimates on bubble graphs. Bubble graphs accommodate gaps in data better than weighted mean box-plots, and the resultant trend lines can be used to estimate the expected prevalence for any given age rather than just the specific age group means. Therefore, bubble graphs are preferable over weighted mean box-plots when considering a disease such as diabetes, for which the steady prevalence increase with age has previously been well established [21]. The trend lines obtained in this study all had high $\mathrm{R}^{2}$ values - specified on each graph for purposes of transparency - indicating that they were representative of the data and took into account a high degree of variance.

Several of this study's findings are in line with previous estimations of diabetes burden in comparable regions. Estimates for 2000 [21] and 2010 [22] both suggest that in developing countries, diabetes burden is highest between the ages of 40 and 64 , and lowest under the age of 40 . This was reflected in the findings. Wild and colleagues also found that global diabetes prevalence was higher overall for males than females. However, more recent estimates for Southern Asia found no distinct increase in diabetes risk with male gender [64]. This study found a small but consistent difference between male and female prevalence. Based on national surveys for countries in the region, Jaywardena and colleagues estimated the overall Southern Asia diabetes prevalence to be in the range of $4.5-10.3 \%$ for the period 1995-2006[64]. This study's estimate of $7.47 \%$ for 2005 falls in the middle of this range. Additionally, this study's 2010 prevalence estimate of $7.60 \%$ is similar to the findings of Shaw and colleagues for the WHO region of South-East Asia[22], a geographic region that includes six out of the nine countries of the UN Southern Asia region (all apart from Afghanistan, Iran and Pakistan). Recent IDF estimations suggest that diabetes prevalence may be even higher - at an estimated $8.60 \%$ for South-East Asia in 2011 [65]. However, it is difficult to make comparisons between estimates for the UN Southern Asia region and the WHO South-East Asia region. In addition, different studies often use widely varying methods for study selection and estimating prevalence, contributing to the observed inconsistencies. Nevertheless, a substantial body of evidence including this study indicate that the diabetes burden in this area of the world is large and growing.

\section{Limitations}

This systematic review considered published studies from 1980-2013. However, suitable studies were only identified for the period 1992-2013. Not looking at studies prior to 1980 may have excluded viable studies, but older studies often used previous diabetes diagnostic criteria which underestimated prevalence. Most of the identified studies carried out prior to 1990 used the old 1980/1985 WHO criteria with the higher FPG cut-off of $7.8 \mathrm{mmol} / \mathrm{L}$ for diabetes diagnosis. Including such studies in the analysis would distort the prevalence estimate because of the differing diabetes case definitions.

Several recent studies did not detail the diagnostic criteria used to identify diabetics, despite explaining their method of diagnosis. These studies were excluded to ensure that the case definition of diabetes in the selected studies remained constant. Other studies did not make it clear whether the biochemical samples they used were venous or capillary, or whether whole blood or plasma was analysed. WHO provides diagnostic guidelines for each of these sample types [11,12], but when sample type was not specified the study was excluded to minimise case definition misclassifications. Adherence to these stringent quality assessment criteria potentially limited the number of studies that could be included in this analysis. In addition, studies that did not diagnose diabetes through biochemical measurements but instead used techniques such as self-reported surveys were also excluded. Several studies had found 
low knowledge of diabetes in Southern Asia, even in diabetic patients $[66,67]$, and therefore such methods were considered unreliable.

Studies investigating other forms of diabetes such as gestational diabetes or diabetes insipidus were excluded. These studies were easy to identify because of the specific nature of gestational diabetes, and the different clinical presentation of diabetes insipidus to diabetes mellitus (DM). However, a major limitation of this paper was the inadequate ability to distinguish between type $1 \mathrm{DM}$ and type $2 \mathrm{DM}$. Many risk factors for type $2 \mathrm{DM}$, such as diet and physical inactivity, are modifiable and therefore may be amenable to policy intervention, but there are no known preventative measures against type $1 \mathrm{DM}$. Most analysed studies did not further investigate identified diabetics to exclude type $1 \mathrm{DM}$, meaning that this study's findings may be an overestimation of the prevalence of type $2 \mathrm{DM}$. However, in the adult age range that was being investigated, type $2 \mathrm{DM}$ is more common than type $1 \mathrm{DM}$, hence their previous names of "adult-onset diabetes mellitus" and "childhood-onset diabetes mellitus". In addition, it has been noted that while type 1 DM rarely causes death by ketoacidosis in developed countries, sufferers in many developing countries may unfortunately have a radically shortened lifespan due to limited insulin availability which is crucial for type $1 \mathrm{DM}$ management [68]. This may hold true especially for some of the poor rural areas investigated in this study. As a result, any error in the prevalence estimate due to type 1 diabetics is likely to be small.

Only published studies were reviewed. The resulting analysis may have suffered from publication bias as the reviewed papers may only show those studies in which significant results were found. Publication bias may have prevented studies that did not show significant diabetes prevalence from being published in the first place, preventing these studies from being captured in this review. While no limits were set on language, time constraints also prevented translation and therefore inclusion of non-English studies. This may have resulted in exclusion of viable nonEnglish studies. However, even without setting language limits, only a very small number of non-English studies were identified by the literature search. This might be due to the status of English as an official language in several excolonial countries in the Southern Asia region, most notably India and Pakistan.

No suitable studies were identified for three out of nine of the countries in the region - Afghanistan, Bhutan, and the Maldives. As mentioned, however, these are the least populous countries in the region, and combined only account for $1.34 \%$ of the regional adult population. Nevertheless it is a noteworthy limitation that no data was available for these countries when the estimated regional population prevalence took their populations into account as well. An- other limitation arose when comparing urban and rural studies. Cohorts were classified as urban or rural on the basis of individual study descriptions. No standardised definitions of 'urban' or 'rural' were used, meaning there may be discrepancies between different studies on their cohort classification.

Not all selected studies provided male and female sex-specific prevalence breakdowns, while others did not provide sample sizes for sex-specific prevalence or did not report mean age. This limited the number of cohorts that could be analysed for male and female age-specific prevalence. Where possible, sample sizes were calculated based on reported number of cases and corresponding prevalence figures. However, this study's findings were limited by assumptions that had to be made to account for incomplete data. UNPD national age structures were applied where appropriate [23]. This method of estimation may have increased imprecision as study populations are not necessarily representative of the national average. The use of a correction factor to account for incomplete sex-specific data may have been another source of imprecision. A hypothetical maximum age of 80 was used to calculate mean age for studies that provided no maximum age range. This assumption was made as this was the highest age used in reviewed studies that included a maximum age, and also because the minority of studies that looked at participants aged 80+ had very small sample sizes for those age groups. In addition, the 2005 UNPD population estimates for Southern Asia suggest that the 80+ age group accounts for only $0.58 \%$ of the population, so this was not considered a major limitation.

While every effort was made to ensure accuracy and the use of systematic methods, human error may have resulted in accidental exclusion of relevant studies when inclusion and quality criteria were being applied. Having only one person review and evaluate studies is a potential limitation of this study design. Using several independent evaluators to select studies, with a suitable method for resolving disputes, may increase reliability of the study's findings.

\section{Implications for policy}

Evidence on disease burden is essential for countries to plan and develop programs in response to the NCD pandemic. The WHO 2008-2013 Action Plan for the Prevention and Control of Noncommunicable Diseases highlighted that before prevention and control policies can be implemented, one of the first steps is to assess the burden of disease [69]. Engelgau and colleagues also proposed a framework for policy decision-making on NCDs[7], and improved surveillance is an essential component of their initial assessment stage. Several Southern Asian countries have shown improvements in their national NCD surveillance and monitoring capabilities in the last decade [2]. 
However, as previously discussed, estimates of diabetes burden vary widely between different sources. Further improvements in national surveillance capabilities are needed so that authoritative and standardised estimations of the burden of NCDs can be made. Accurate and up-to-date estimations of burden are also important in evaluation of current policies, programs, and of health system capacity. A number of policies have shown promise - in 2003 India enacted The Cigarette and Other Tobacco Products Act which is considered to have effectively reduced the public's exposure to tobacco smoke, through advertisement, and minors' access to cigarettes [7]. India also recently launched a pilot phase of the National Programme for Prevention and Control of Diabetes, Cardiovascular Diseases and Stroke (NPDCS), and has made financing commitments for the near future [70]. The aims of this program are laudable but monitoring of such programmes is necessary to ensure resources are used efficiently, and that appropriate targets and priorities are set [7]. This is of special importance due to rapidly-changing nature of NCDs and the many challenges that governments of developing Southern Asian countries face when attempting to deal with them.

The findings of this study suggest that future urbanisation and increased life expectancy will lead to a substantial rise in the burden of type 2 DM in the Southern Asia region. Commentators have noted that the process of population ageing currently being observed in developing countries is different to the demographic transition that occurred in developed countries several decades ago. In particular, the current demographic transition in developing countries has occurred on a faster scale than in developed countries, and without the associated improvements in living conditions, social provisions, and access to health care [7]. This has led to a 'compressed timeline' for developing countries to mount effective responses to growing NCD burdens compared to what developed countries had [1]. 'Unhealthy ageing' in Southern Asia due to these lagging improvements in nutrition and socioeconomic conditions is predicted to add to the natural increases in disease burden expected of an ageing population [7]. Therefore it is paramount that both prevention and treatment policy options are considered - the root causes of NCDs need to be addressed, and health system capacity should be reviewed to deal with the increasing burden.

Prevention efforts for diabetes can come in many forms, but there are several that may be especially relevant to Southern Asia. Before any successful prevention policies can be implemented it is important that knowledge and awareness regarding diabetes is improved in the general population. Many studies have shown that in Southern Asian populations, awareness of diabetes and its risk factors is poor $[71,72]$, even among diabetic patients $[66,67]$. Populationlevel education and health promotion schemes should be put into place to improve awareness of the risk factors for diabetes and the other main NCDs. Diabetes has many lifestyle-modifiable risk factors, and by improving knowledge regarding these, the Southern Asian population can be empowered to pursue healthier lifestyle choices. Increased risk factor awareness and the resulting community empowerment have been seen to have a positive effect in the past. Mohan and colleagues reported that following such efforts, an Indian community was prompted to create a public park with their own funds which resulted in significant improvements in physical activity levels for local resident [73].

Departure from traditional dietary patterns and the uptake of diets high in saturated fats and heavily refined carbohydrates are believed to be important underlying factors in rising rates of obesity and diabetes in Southern Asia [17]. In particular, low intake of fibre, mono- and poly-unsaturated fats, and high consumption of refined carbohydrates, saturated fats and trans-fats have been identified as problematic dietary habits leading to insulin resistance in Southern Asian populations [74]. Policies should focus on addressing these unhealthy dietary patterns with a view to inform and educate. Successful policies from the health sector may include efforts to improve food labelling through dialogue with food companies, which when combined with education on NCD dietary risk factors may go some way to lowering diabetes and obesity incidence [7]. Focus should also be given on encouraging people to switch from traditional high trans-fat cooking oils such as ghee and vanaspati to poly-unsaturated alternatives [74].

The health sector has an important role to play in the management and treatment of NCDs. Table 2 shows that while all the Southern Asian economies are growing, many of them spend very small proportions of their gross domestic product (GDP) as health expenditure. In 2001, the WHO Commission on Macroeconomics and Health found that a basic set of essential health interventions costs approximately US $\$ 34$ per capita, believed to be a modest sum even for low-income countries [75]. However, several Southern Asian countries spend less on health than even this recommended minimum per capita expenditure [20]. NCDs undermine economic progress and have significant macroeconomic and microeconomic impacts [76]. The increasing burden of NCDs will strain existing health systems if health expenditure is not increased. Physician density is also low in many Southern Asian countries; Engelgau and colleagues suggest that improving region-wide health education and training capacities may help fill human resource gaps across the region by taking advantage of economies of scale [7].

Even if health system capacities are expanded to deal with the increasing burden of NCDs, access to appropriate health care remains a major challenge across the Southern Asia region. Studies in several Southern Asian countries have found significant personal expenditure and substan- 
tial financial loss associated with paying for diabetes treatment, with the main costs being the direct expenses of investigation, treatment, and hospital admission $[49,77,78]$. These expensive out-of-pocket medical costs are a major barrier for universal access to health care services in Southern Asia, and result in widening inequalities between rich and poor. In addition to increasing health care capacity, Southern Asian countries should aim to improve access to health care by implementing WHO universal coverage reforms [79]. Successful policy strategies may involve improving revenue collection by targeting tax avoidance; pooling risk to reduce cost-sharing; efficiency savings from introducing Health Technologies Assessment (HTA); and simply increasing the priority given to health and thereby increasing governmental health expenditure.

\section{Implications for future research}

Following the recent WHO addendum approving the use of $\mathrm{HbAl}_{\mathrm{c}}$ as a diagnostic method [1], WHO and American Diabetes Association diagnostic guidelines and criteria are mostly aligned. Future studies investigating prevalence in a population or community should use these standardised methods and criteria for diagnosing diabetes to allow for informative comparison between studies. The utilisation of $\mathrm{HbAl}_{\mathrm{c}}$ measurements also presents new avenues for diabetes epidemiological research. If appropriate quality assurance measures are put in place, as per WHO recommendations, $\mathrm{HbAl}{ }_{\mathrm{c}}$ presents a valuable method for investigating long term changes in glycaemic status in study subjects.

Studies calculating overall prevalence inadvertently calculate male and female prevalence as well, but as seen in this paper, in many cases these were not reported. Additionally many studies failed to report basic information such as mean age of sample, age group of sample, type of biochemical sample taken, or diagnostic criteria used to define diabetes. Access to this information would reduce the number of assumptions that have to be made for incomplete data, and additionally would allow for a more accurate estimate as far fewer studies would have to be excluded from analysis. As awareness of the need for large-scale population estimates of NCDs becomes more commonplace, it is hoped authors carrying out community-based studies begin to employ common standards to allow effective utilisation of their work in burden of disease analyses.

Diabetes is one end of a spectrum of glycaemic states, and future studies could estimate the burden of different forms of prediabetes as well. Impaired fasting glucose (IFG) and impaired glucose tolerance (IGT) are diagnosed with FPG and OGTT respectively so many studies report their prevalence alongside diabetes. Regional estimation of the burden of both prediabetes as well as diabetes would allow for a more comprehensive analysis of the challenges these hyperglycaemic diseases pose.
This study found that diabetes is strongly associated with urban residency in Southern Asia. As the region continues a process of rapid globalisation and urbanisation, it is important to maintain diabetes surveillance in both urban and rural cohorts. Urban migration and the increasing accessibility of inactive, sedentary lifestyles suggest that the diabetes burden will increase in the future. Projections for 2030 predict that several Southern Asian nations will continue to rank among the countries with the largest numbers of diabetic residents [21,22]. However, monitoring and analysis of these vulnerable populations can inform public health policymakers and help manage the burden of diabetes. It is important that future studies focus on high-risk populations: urban residents, and, as life expectancy increases, the growing number of elderly people as well.

\section{CONCLUSION}

This systematic literature review found a high prevalence of type $2 \mathrm{DM}$ in the Southern Asia region. Diabetes prevalence was associated with male gender, and strongly associated with older age and urban residency. On the basis of these findings this study also predicted that diabetes prevalence will continue to increase in the future as life expectancy in the region rises and countries continue to undergo processes of urbanisation. The findings of this study were consistent with several past studies, but dissimilar to the results of others. This highlights that inconsistent surveillance and conflicting estimations of burden are some of the many challenges faced by the Southern Asia region and its constituent countries in their effort to respond to the rising burden of NCDs.

Accurate and up-to-date estimates of burden of disease are essential for planning of policies, target and priority setting, as well as monitoring and assessment of existing programs. However, greater standardisation and shared principles are needed across different studies so that strong, clear messages are given to policymakers. It is hoped that improved surveillance capabilities in Southern Asian countries will encourage common standards for prevalence estimation to be established.

While current policies and programs on diabetes control have met with some success, the region faces numerous hurdles. Rising life expectancies coupled with 'unhealthy' ageing present a new set of challenges to those faced by developed countries several decades ago. Policies focusing on prevention have to deal with a population that largely has little awareness of diabetes and its risk factors, and is becoming increasingly accustomed to a sedentary lifestyle and unhealthy eating patterns. Health sectors also have their own set of issues - total health expenditure is low in many Southern Asian countries, there are significant hu- 
man resource gaps, and already struggling health systems are predicted to be put under even greater strain as diabetes prevalence continues to increase. In addition, from an equity perspective, high cost-sharing coupled with the long-term care needed for NCDs such as diabetes means that access to health care may be limited for a large proportion of people in the region.

However, despite these numerous policy challenges and the projected increase in diabetes prevalence, slow progress is being made. NCDs are at the forefront of the international health agenda, several Southern Asian countries have greatly improved their NCD surveillance and monitoring capacities, and the numbers of studies estimating burden of type 2 diabetes mellitus appears to be increasing in recent years. Greater attention needs to be paid to this disease and its risk factors on national and regional levels in Southern Asia so that the growing burden of diabetes can be adequately addressed in the future.

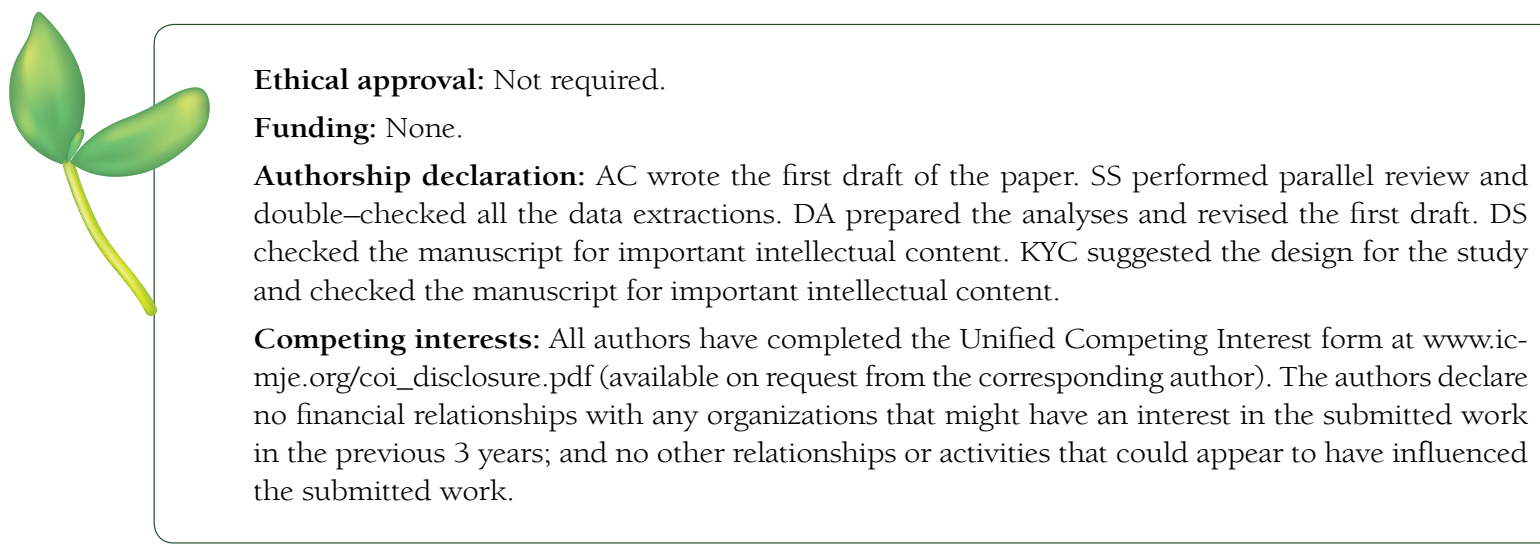

1 World Health Organization. UN High Level Meeting on NCDS: Summary report of the discussions at the round tables. Geneva: WHO, 2011. Available at: http://www.who.int/nmh/events/moscow_ncds_2011/round_tables_ summary.pdf.Accessed: 22 January 2013.

2 Alwan A, MacLean DR, Riley LM, d'Espaignet ET, Mathers CD, Stevens GA, et al. Monitoring and surveillance of chronic non-communicable diseases: progress and capacity in high-burden countries. Lancet. 2010;376:18618. Medline:21074258 doi:10.1016/S0140-6736(10)61853-3

3 United Nations. Prevention and control of non-communicable diseases: Report of the Secretary-General. New York: United Nations, 2011. Available at: http://www.un.org/ga/search/view_doc.asp?symbol=A/66/83\&Lang=E. Accessed: 22 January 2013.

4 Boutayeb A. The double burden of communicable and non-communicable diseases in developing countries. Trans R Soc Trop Med Hyg. 2006;100:191-9. Medline:16274715 doi:10.1016/j.trstmh.2005.07.021

5 Beaglehole R, Bonita R, Horton R, Adams C, Alleyne G, Asaria P, et al. Priority actions for the non-communicable disease crisis. Lancet. 2011;377:1438-47. Medline:21474174 doi:10.1016/S0140-6736(11)60393-0

6 World Health Organization. Commission on the Social Determinants of Health. Geneva: WHO, 2008.

7 Engelgau MM, El-Saharty S, Kudesia P, Rajan V, Rosenhouse S, Okamoto K. Capitalizing on the demographic transition: tackling noncommunicable diseases in South Asia. Washington DC: The World Bank, 2011.

8 Miranda JJ, Kinra S, Casas JP, Davey Smith G, Ebrahim S. Non-communicable diseases in low- and middle-income countries: context, determinants and health policy. Trop Med Int Health. 2008;13:1225-34. Medline:18937743 doi:10.1111/j.1365-3156.2008.02116.x

9 Chan KY, Adeloye D, Grant L, Kolčić I, Marušić A. Estimating the burden of non-communicable diseases in low- and middle-income countries. J Glob Health. 2012;2:020101. Medline:23289068

10 American Diabetes Association. Diagnosis and classification of diabetes mellitus. Diabetes Care. 2010;33:S62-9. Medline:20042775 doi:10.2337/dc10-S062

11 World Health Organization. Definition and diagnosis of diabetes mellitus and intermediate hyperglycaemia: Report of a WHO/IDF Consultation. Geneva: WHO, 2006.

12 World Health Organization. Definition, diagnosis and classification of diabetes mellitus and its complications. Geneva: WHO Department of Non-Communicable Disease Surveillance, 1999.

13 World Health Organization. use of glycatedhaemoglobin (hbalc) in the diagnosis of diabetes mellitus: Abbreviated Report of a WHO Consultation. Geneva: WHO, 2011.

14 Mokdad AH, Ford ES, Bowman BA, Dietz WH, Vinicor F, Bales VS, et al. Prevalence of obesity, diabetes, and obesity-related health risk factors, 2001. JAMA. 2003;289:76-9. Medline:12503980 doi:10.1001/jama.289.1.76 
15 Wang J, Luben R, Khaw K, Bingham S, Wareham NJ, Forouhi NG. Dietary energy density predicts the risk of incident type 2 diabetes. Diabetes Care. 2008;31:2120-5. Medline:18689693 doi:10.2337/dc08-1085

16 Expert Consultation WHO. Appropriate body-mass index for Asian populations and its implications for policy and intervention strategies. Lancet. 2004;363:157-63. Medline:14726171 doi:10.1016/S0140-6736(03)15268-3

17 Mohan V. Why are Indians more prone to diabetes? J Assoc Physicians India. 2004;52:468-74. Medline:15645957

18 Misra A, Vikram NK. Insulin resistance syndrome (metabolic syndrome) and obesity in Asian Indians: Evidence and implications. Nutrition. 2004;20:482-91. Medline:15105039 doi:10.1016/j.nut.2004.01.020

19 United Nations. Composition of macro geographical (continental) regions, geographical sub-regions, and selected economic other groupings. New York: United Nations Statistics Division, 2013. Available at: http://unstats.un.org/unsd/methods/m49/m49regin.htm. Accessed: 22 January 2013.

20 World Bank. Countries and Economies. The World Bank Group, 2013. Available at: http://data.worldbank.org/ country. Accessed: 6 February 2013.

21 Wild S, Roglic G, Green A, Sicree R, King H. Global prevalence of diabetes: estimates for the year 2000 and projections for 2030. Diabetes Care. 2004;27:1047-53. Medline:15111519 doi:10.2337/diacare.27.5.1047

22 Shaw JE, Sicree RA, Zimmet PZ. Global estimates of the prevalence of diabetes for 2010 and 2030. Diabetes Res Clin Pract. 2010;87:4-14. Medline:19896746 doi:10.1016/j.diabres.2009.10.007

23 United Nations Population Division. World population prospects, the 2010 Revision. New York: United Nations Department of Economic and Social Affairs, Population Division, Population Estimates and Projections Section, 2011. Available at: http://esa.un.org/unpd/wpp/Excel-Data/population.htm. Accessed: 12 February 2013.

24 Bhowmik B, Binte Munir S, Ara Hossain I, Siddiquee T, Diep LM, Mahmood S, et al. Prevalence of type 2 diabetes and impaired glucose regulation with associated cardiometabolic risk factors and depression in an urbanizing rural community in Bangladesh: a population-based cross-sectional study. Diabetes Metab J. 2012;36:42232. Medline:23275936 doi:10.4093/dmj.2012.36.6.422

25 Rahim MA, Khan AK, Ali SM, Nahar Q, Shaheen A, Hussain A. Glucose tolerance in a rural population of Bangladesh. Int J Diabetes Dev Ctries. 2008;28:45-50. Medline:19902047 doi:10.4103/0973-3930.43098

26 Nazir A, Papita R, Anbalagan VP, Anjana RM, Deepa M, Mohan V. Prevalence of diabetes in Asian Indians based on glycated hemoglobin and fasting and 2-H post-load (75-g) plasma glucose (CURES-120). Diabetes Technol Ther. 2012;14:665-8. Medline:22823754 doi:10.1089/dia.2012.0059

27 Prasad DS, Kabir Z, Dash AK, Das BC. Prevalence and risk factors for diabetes and impaired glucose tolerance in Asian Indians: a community survey from urban eastern India. Diabetes Metab Syndr. 2012;6:96-101. Medline:23153977 doi:10.1016/j.dsx.2012.05.016

28 Anjana RM, Pradeepa R, Deepa M, Datta M, Sudha V, Unnikrishnan R, et al. Prevalence of diabetes and prediabetes (impaired fasting glucose and/or impaired glucose tolerance) in urban and rural India: phase I results of the Indian Council of Medical Research-INdiaDIABetes (ICMR-INDIAB) study. Diabetologia. 2011;54:3022-7. Medline:21959957 doi:10.1007/s00125-011-2291-5

29 Deepa M, Anjana RM, Manjula D, Narayan KM, Mohan V. Convergence of prevalence rates of diabetes and cardiometabolic risk factors in middle and low income groups in urban India: 10-year follow-up of the Chennai Urban Population Study. J Diabetes Sci Techol. 2011;5:918-27. Medline:21880235 doi:10.1177/193229681100500415

30 Ravikumar P, Bhansali A, Ravikiran M, Bhansali S, Walia R, Shanmugasundar G, et al. Prevalence and risk factors of diabetes in a community-based study in North India: the Chandigarh Urban Diabetes Study (CUDS). Diabetes Metab. 2011;37:216-21. Medline:21195002 doi:10.1016/j.diabet.2010.10.004

31 Ramachandran A, Mary S, Yamuna A, Murugesan N, Snehalatha C. High prevalence of diabetes and cardiovascular risk factors associated with urbanization in India. Diabetes Care. 2008;31:893-8. Medline:18310309 doi:10.2337/dc07-1207

32 Zargar AH, Wani AA, Laway BA, Masoodi SR, Wani AI, Bashir MI, et al. Prevalence of diabetes mellitus and other abnormalities of glucose tolerance in young adults aged 20-40 years in North India (Kashmir Valley). Diabetes Res Clin Pract. 2008;82:276-81. Medline:18814927 doi:10.1016/j.diabres.2008.08.006

33 Sadikot SM, Nigam A, Das S, Bajaj S, Zargar AH, Prasannakumar KM, et al. The burden of diabetes and impaired glucose tolerance in India using the WHO 1999 criteria: prevalence of diabetes in India study (PODIS). Diabetes Res Clin Pract. 2004;66:301-7. Medline:15609460 doi:10.1016/j.diabres.2004.04.008

34 Ramachandran A, Snehalatha C, Kapur A, Vijay V, Mohan V, Das AK, et al. High prevalence of diabetes and impaired glucose tolerance in India: National Urban Diabetes Survey. Diabetologia. 2001;44:1094-101. Medline:11596662 doi:10.1007/s001250100627

35 Hadaegh F, Bozorgmanesh MR, Ghasemi A, Harati H, Saadat N, Azizi F. High prevalence of undiagnosed diabetes and abnormal glucose tolerance in the Iranian urban population: Tehran Lipid and Glucose Study. BMC Public Health. 2008;8:176. Medline:18501007 doi:10.1186/1471-2458-8-176

36 Sadeghi M, Roohafza H, Shirani S, Poormoghadas M, Kelishadi R, Baghaii A, et al. Diabetes and associated cardiovascular risk factors in Iran: the Isfahan Healthy Heart Programme. Ann Acad Med Singapore. 2007;36:17580. Medline: 17450262

37 Shrestha UK, Singh DL, Bhattarai MD. The prevalence of hypertension and diabetes defined by fasting and 2-h plasma glucose criteria in urban Nepal. Diabet Med. 2006;23:1130-5. Medline:16978379 doi:10.1111/ j.1464-5491.2006.01953.x 
38 Katulanda P, Constantine GR, Mahesh JG, Sheriff R, Seneviratne RD, Wijeratne S, et al. Prevalence and projections of diabetes and pre-diabetes in adults in Sri Lanka-Sri Lanka Diabetes, Cardiovascular Study (SLDCS). Diabet Med. 2008;25:1062-9. Medline:19183311 doi:10.1111/j.1464-5491.2008.02523.x

39 Rahman MM, Rahim MA, Nahar Q. Prevalence and risk factors of type 2 diabetes in an urbanizing rural community of Bangladesh. Bangladesh Med Res Counc Bull. 2007;33:48-54. Medline:18481438

40 Hussain A, Rahim MA, Azad Khan AK, Ali SM, Vaaler S. Type 2 diabetes in rural and urban population: diverse prevalence and associated risk factors in Bangladesh. Diabet Med. 2005;22:931-6. Medline:15975110 doi:10.1111/j.1464-5491.2005.01558.x

41 Pandey RM, Gupta R, Misra A, Misra P, Singh V, Agrawal A, et al. Determinants of urban-rural differences in cardiovascular risk factors in middle-aged women in India: a cross-sectional study. Int J Cardiol. 2013;163:157-62. Medline:21880382 doi:10.1016/j.ijcard.2011.06.008

42 Vaz NC, Ferreira AM, Kulkarni MS, Vaz FS. Prevalence of diabetes mellitus in a rural population of Goa, India. Natl Med J India. 2011;24:16-8. Medline:21608352

43 Rao CR, Kamath VG, Shetty A, Kamath A. A study on the prevalence of type 2 diabetes in coastal Karnataka. Int J Diabetes Dev Ctries. 2010;30:80-5. Medline:20535311 doi:10.4103/0973-3930.62597

44 Vijayakumar G, Arun R, Kutty VR. High prevalence of type 2 diabetes mellitus and other metabolic disorders in rural Central Kerala. J Assoc Physicians India. 2009;57:563-7. Medline:20209716

45 Namperumalsamy P, Kim R, Vignesh TP, Nithya N, Royes J, Gijo T, et al. Prevalence and risk factors for diabetic retinopathy: a population-based assessment from Theni District, south India. Br J Ophthalmol. 2009;93:42934. Medline: 19091856

46 Chow CK, Raju PK, Raju R, Reddy KS, Cardona M, Celermajer DS, et al. The prevalence and management of diabetes in rural India. Diabetes Care. 2006;29:1717-8. Medline:16801618 doi:10.2337/dc06-0621

47 Gupta A, Gupta R, Sarna M, Rastogi S, Gupta VP, Kothari K. Prevalence of diabetes, impaired fasting glucose and insulin resistance syndrome in an urban Indian population. Diabetes Res Clin Pract. 2003;61:69-76. Medline:12849925 doi:10.1016/S0168-8227(03)00085-8

48 Misra A, Pandey RM, Devi JR, Sharma R, Vikram NK, Khanna N. High prevalence of diabetes, obesity and dyslipidaemia in urban slum population in northern India. Int J Obes Relat Metab Disord. 2001;25:1722-9. Medline:11753596 doi:10.1038/sj.ijo.0801748

49 Esteghamati A, Khalilzadeh O, Anvari M, Meysamie A, Abbasi M, Forouzanfar M, et al. The economic costs of diabetes: a population-based study in Tehran, Iran. Diabetologia. 2009;52:1520-7. Medline:19475364 doi:10.1007/s00125-009-1398-4

50 Azimi-Nezhad M, Ghayour-Mobarhan M, Parizadeh MR, Safarian M, Esmaeili H, Parizadeh SM, et al. Prevalence of type 2 diabetes mellitus in Iran and its relationship with gender, urbanisation, education, marital status and occupation. Singapore Med J. 2008;49:571-6. Medline:18695867

51 Sharma SK, Ghimire A, Radhakrishnan J, Thapa L, Shrestha NR, Paudel N, et al. Prevalence of hypertension, obesity, diabetes, and metabolic syndrome in Nepal of type 2 diabetes mellitus. Int J Hypertens . 2011;2011:821971. Medline:21629873 doi:10.4061/2011/821971

52 Paudyal G, Shrestha MK, Meyer JJ, Thapa R, Gurung R, Ruit S. Prevalence of diabetic retinopathy following a community screening for diabetes. Nepal Med Coll J. 2008;10:160-3. Medline:19253859

53 Basit A. Danish Alvi SF, Fawwad A, Ahmed K, Yakoob Ahmedani M, Hakeem R. Temporal changes in the prevalence of diabetes, impaired fasting glucose and its associated risk factors in the rural area of Baluchistan. Diabetes Res Clin Pract. 2011;94:456-62. Medline:21890227 doi:10.1016/j.diabres.2011.08.009

54 Zafar J, Bhatti F, Akhtar N, Rasheed U, Bashir R, Humayun S, et al. Prevalence and risk factors for diabetes mellitus in a selected urban population of a city in Punjab. J Pak Med Assoc. 2011;61:40-7. Medline:22368901

55 Mahar PS, Awan MZ, Manzar N, Memon MS. Prevalence of type-II diabetes mellitus and diabetic retinopathy: the Gaddap study. J Coll Physicians Surg Pak. 2010;20:528-32. Medline:20688018

56 Basit A, Hydrie MZ, Ahmed K, Hakeem R. Prevalence of diabetes, impaired fasting glucose and associated risk factors in a rural area of Baluchistan province according to new ADA criteria. J Pak Med Assoc. 2002;52:35760. Medline: 12481676

57 Pinidiyapathirage MJ, Kasturiratne A, Ranawaka UK, Gunasekara D, Wijekoon N, Medagoda K, et al. The burden of diabetes mellitus and impaired fasting glucose in an urban population of Sri Lanka. Diabet Med. 2013;30:326-32. Medline:22998091 doi:10.1111/dme.12013

58 Wijewardene K, Mohideen MR, Mendis S, Fernando DS, Kulathilaka T, Weerasekara D, et al. Prevalence of hypertension, diabetes and obesity: baseline findings of a population based survey in four provinces in Sri Lanka. Ceylon Med J. 2005;50:62-70. Medline:16114771

59 Boddula R, Yadav S, Bhatia V, Genitta G, Pandey D, Kumar A, et al. High prevalence of type 2 diabetes mellitus in affluent urban Indians. Diabetes Res Clin Pract. 2008;81:e4-7. Medline:18486256 doi:10.1016/j.diabres.2008.04.003

60 Ramachandran A, Snehalatha C, Baskar AD, Mary S, Kumar CK, Selvam S, et al. Temporal changes in prevalence of diabetes and impaired glucose tolerance associated with lifestyle transition occurring in the rural population in India. Diabetologia. 2004;47:860-5. Medline:15114469 doi:10.1007/s00125-004-1387-6

61 Ramachandran A, Snehalatha C, Shyamala P, Vijay V, Viswanathan M. High prevalence of NIDDM and IGT in an elderly south Indian population with low rates of obesity. Diabetes Care. 1994;17:1190-2. Medline:7821141 doi:10.2337/diacare.17.10.1190 
62 Ramachandran A, Snehalatha C, Dharmaraj D, Viswanathan M. Prevalence of glucose intolerance in Asian Indians. Urban-rural difference and significance of upper body adiposity. Diabetes Care. 1992;15:1348-55. Medline: 1425100 doi:10.2337/diacare.15.10.1348

63 King H, Aubert RE, Herman WH. Global Burden of Diabetes, 1995-2025: Prevalence, numerical estimates, and projections. Diabetes Care. 1998;21:1414-31. Medline:9727886 doi:10.2337/diacare.21.9.1414

64 Jayawardena R, Ranasinghe P, Byrne NM, Soares MJ, Katulanda P, Hills AP. Prevalence and trends of the diabetic epidemic in South Asia: a systematic review and meta-analysis. BMC Public Health. 2012;12:380. Medline:22630043 doi:10.1186/1471-2458-12-380

65 Whiting DR, Guariguata L, Weil C, Shaw J. IDF Diabetes Atlas: Global estimates of the prevalence of diabetes for 2011 and 2030. Diabetes Res Clin Pract. 2011;94:311-21. Medline:22079683 doi:10.1016/j.diabres.2011.10.029

66 Murugesan N, Snehalatha C, Shobhana R, Roglic G, Ramachandran A. Awareness about diabetes and its complications in the general and diabetic population in a city in southern India. Diabetes Res Clin Pract. 2007;77:4337. Medline:17291622 doi:10.1016/j.diabres.2007.01.004

67 Gul N. Knowledge, attitudes and practices of type 2 diabetic patients. J Ayub Med Coll Abbottabad. 2010;22:12831. Medline:22338437

68 Yudkin JS. Insulin for the world's poorest countries. Lancet. 2000;355:919-21. Medline:10752719 doi:10.1016/ S0140-6736(99)09225-9

69 World Health Organization. 2008-2013 Action plan for the global strategy for the prevention and control of noncommunicable diseases. Geneva: WHO, 2008.

70 Verma R, Khanna P, Mehta B. National programme on prevention and control of diabetes in India: Need to focus. Australas Med J. 2012;5:310-5. Medline:22848329 doi:10.4066/AMJ.2012.1340

71 Rafique G, Azam SI, White F. Diabetes knowledge, beliefs and practices among people with diabetes attending a university hospital in Karachi, Pakistan. East Mediterr Health J. 2006;12:590-8. Medline:17333798

72 Ulvi OS, Chaudhary RY, Ali T, Alvi RA, Khan MF, Khan M, et al. Investigating the awareness level about diabetes mellitus and associated factors in Tarlai (Rural Islamabad). J Pak Med Assoc. 2009;59:798-801. Medline:20361687

73 Mohan V, Shanthirani CS, Deepa M, Datta M, Williams OD, Deepa R. Community empowerment - a successful model for prevention of non-communicable diseases in India - the Chennai Urban Population Study (CUPS17). J Assoc Physicians India. 2006;54:858-62. Medline:17249253

74 Misra A, Khurana L, Isharwal S, Bhardwaj S. South Asian diets and insulin resistance. Br J Nutr. 2009;101:46573. Medline: 18842159 doi:10.1017/S0007114508073649

75 World Health Organization. Macroeconomics and health: Investing in health for economic development. Geneva: WHO, 2001.

76 Mahal A, Karan A, Engelgau M. the economic implications of non-communicable disease for India. Washington DC: The World Bank, 2010.

77 Khowaja LA, Khuwaja AK, Cosgrove P. Cost of diabetes care in out-patient clinics of Karachi, Pakistan. BMC Health Serv Res. 2007;7:189. Medline:18028552 doi:10.1186/1472-6963-7-189

78 Tharkar S, Devarajan A, Kumpatla S, Viswanathan V. The socioeconomics of diabetes from a developing country: A population based cost of illness study. Diabetes Res Clin Pract. 2010;89:334-40. Medline:20538363 doi:10.1016/j.diabres.2010.05.009

79 World Health Organization. The World Health Report 2010: Health systems financing - The path to universal coverage. Geneva: WHO, 2010. 